\title{
Anticancer and Antifungal Activity of Copper(II) Complexes of Quinolin-2(1H)-one-Derived Schiff Bases
}

\author{
Bernadette S. Creaven \\ Technological University Dublin \\ Brian Duff \\ Technological University Dublin \\ Denise A. Egan \\ Technological University Dublin
}

See next page for additional authors

Follow this and additional works at: https://arrow.tudublin.ie/ittsciart

Part of the Medicinal Chemistry and Pharmaceutics Commons, and the Pharmacology Commons

\section{Recommended Citation}

Creaven, B. et al. Anticancer and antifungal activity of copper(II) complexes of quinolin-2(1H)-one-derived Schiff bases. Inorganica Chimica Acta, 363 (2010) 4048-4058 doi:10.1016/j.ica.2010.08.009

This Article is brought to you for free and open access by the School of Science and Computing at ARROW@TU Dublin. It has been accepted for inclusion in Articles by an authorized administrator of ARROW@TU Dublin. For more information, please contact

arrow.admin@tudublin.ie, aisling.coyne@tudublin.ie, gerard.connolly@tudublin.ie.

Funder: Institute of Technology Tallaght

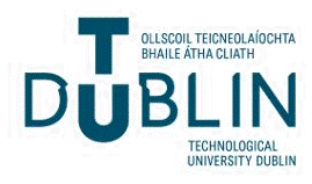




\section{Authors}

Bernadette S. Creaven, Brian Duff, Denise A. Egan, Kevin Kavanagh, Georgina Rosair, Venkat Reddy Thangella, and Maureen Walsh 
Provided for non-commercial research and education use. Not for reproduction, distribution or commercial use.

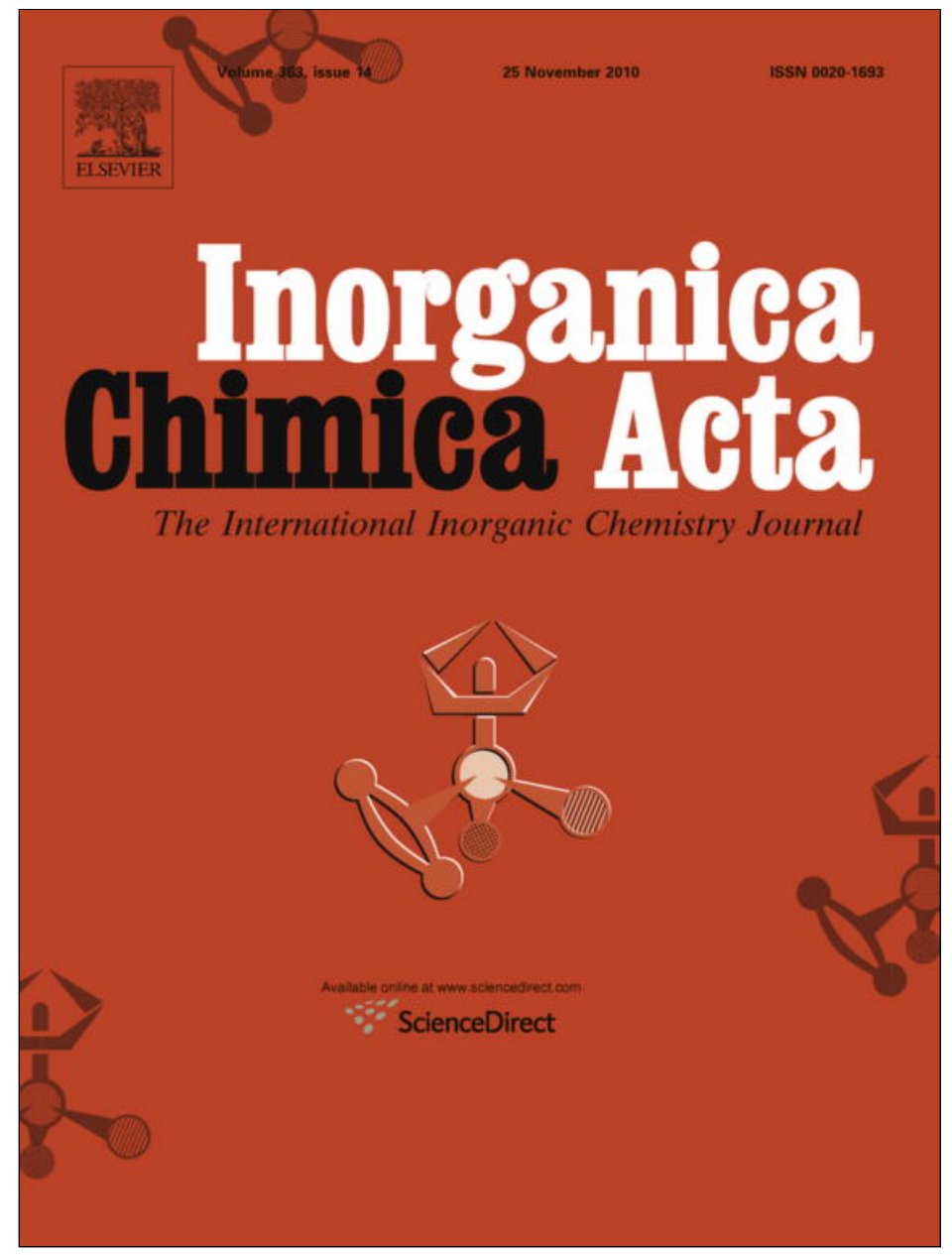

This article appeared in a journal published by Elsevier. The attached copy is furnished to the author for internal non-commercial research and education use, including for instruction at the authors institution and sharing with colleagues.

Other uses, including reproduction and distribution, or selling or licensing copies, or posting to personal, institutional or third party websites are prohibited.

In most cases authors are permitted to post their version of the article (e.g. in Word or Tex form) to their personal website or institutional repository. Authors requiring further information regarding Elsevier's archiving and manuscript policies are encouraged to visit:

http://www.elsevier.com/copyright 


\title{
Anticancer and antifungal activity of copper(II) complexes of quinolin-2 $(1 H)$-one-derived Schiff bases
}

\author{
Bernadette S. Creaven ${ }^{\mathrm{a}, \mathrm{b}, *}$, Brian Duff ${ }^{\mathrm{a}, \mathrm{b}}$, Denise A. Egan ${ }^{\mathrm{a}, \mathrm{b}}$, Kevin Kavanagh ${ }^{\mathrm{c}}$, Georgina Rosair ${ }^{\mathrm{d}}$, \\ Venkat Reddy Thangella ${ }^{\mathrm{a}, \mathrm{b}}$, Maureen Walsh ${ }^{\mathrm{a}, \mathrm{b}}$ \\ ${ }^{a}$ Department of Science, Institute of Technology Tallaght, Dublin 24, Ireland \\ ${ }^{\mathrm{b}}$ Centre for Applied Science and Health, Institute of Technology Tallaght, Dublin 24, Ireland \\ ${ }^{\mathrm{c}}$ Department of Biology, National University of Ireland, Maynooth Co., Kildare, Ireland \\ ${ }^{\mathrm{d}}$ Chemistry, School of Engineering and Physical Science, Heriot Watt University, UK
}

\section{A R T I C L E I N F O}

\section{Article history:}

Received 23 June 2010

Accepted 4 August 2010

Available online 10 August 2010

\section{Keywords:}

Quinolin-2(1H)-one

Schiff bases

Copper(II) complexes

Biological activity

$\mathrm{X}$-ray structures

\section{Introduction}

Antimicrobial drug resistance is of increasing importance as the phenomenon has considerable impact on human and animal health. The prevalence of clinical drug resistance has unfortunately increased significantly in recent decades due to the use and misuse of antimicrobial drugs and many infectious diseases can no longer be treated effectively with common anti-infective drugs. Antimicrobial resistance is not limited to bacterial species and in the 1990s resistance emerged to one of the most potent antifungal agents, fluconazole [1]. Antifungal resistance is particularly problematic as diagnosis is often delayed and there are relatively few antifungal treatments approved for use by the FDA. Over the last number of years, our research group has been interested in developing compounds which have anti-Candida activity as Candida albicans is associated with fungal infections that typically occur in severely immunocompromised and/or critically ill patients [2].

* Corresponding author at: Department of Science, Institute of Technology Tallaght, Dublin 24, Ireland.

E-mail address: bernie.creaven@ittdublin.ie (B.S. Creaven).
Quinolinones, an important class of heterocyclic compounds are part of the quinoline alkaloid family and are also known for their diverse biological activity [3]. Quinolinone heterocycles have emerged as potential therapeutic agents and are the basis of many medicinal drugs used in the treatment of heart failure, cancer and inflammatory diseases [4]. They are also used as antibacterial, antifungal, anti-inflammatory, antitubercular, anticancer, antineoplastic, anti-ischemic, anti-allergic, antihypertensive and antiulcerative agents [5-9]. Recently, a novel series of quinolinones have shown potent inhibitory activity against human immunodeficiency virus type-1 (HIV-1) virus and also exhibited promising activity against several non-nucleoside reverse transcriptase inhibitors (NNRTIs) [10]. One of the 6-functionalised quinolinone derivatives was also reported to possess potent antitumor activity and is undergoing human phase II/III clinical trails as an orally active antitumor drug for the treatment of haematological and solid tumours in the nanomolar range [8].

Schiff bases of azomethine nitrogen donor heterocyclic ligands are well known due to their wide range of applications in pharmaceutical and industrial fields [11]. Transition metal complexes of Schiff bases have attracted a lot of interest due to their potent biological activities such as antifungal, antibacterial, anticancer and herbicidal applications [12-16]. These studies have shown that 
complexation of metals to Schiff base ligands improves the antimicrobial and anticancer activities of the ligands [12]. In addition, more recently reports of quinolin-2(1H)-one-derived Schiff bases and their metal complexes have emerged [17-22]. Several Schiff base ligands derived from pyridine derivatives and their copper(II) complexes have also been shown to inhibit tumour growth [23].

Our previous work has focussed on the synthesis and therapeutic applications of a series of silver and copper complexes of coumarin-derived ligands [24-29]. More recently we have focused our research efforts on the preparation of a series of coumarinand quinolin-2(1H)-one-triazole-derived Schiff bases and their $\mathrm{Cu}(\mathrm{II})$ complexes [30,31]. In this paper, we report a new series of quinolin-2(1H)-one derived Schiff base ligands and their copper complexes and the assessment of their antimicrobial activities against $C$. albicans, and against Gram-negative and Gram-positive bacterial species. Our earlier studies had indicated that coumarin-derived copper(II) complexes had promising anticancer activity. Consequently, in the current study we have investigated the activity of a number of novel copper(II) complexes using the human-derived hepatic carcinoma cell line, Hep- $\mathrm{G}_{2}$.

\section{Experimental}

\subsection{Materials/instrumentation}

All chemicals were purchased from Sigma-Aldrich were reagent grade and used without further purification. Microanalytical data were provided by the Microanalytical Laboratory, National University of Ireland, Belfield, and Dublin 4. Infrared spectra were recorded in the region of $4000-400 \mathrm{~cm}^{-1}$, on a Nicolet Impact 410 Fourier-transform infrared (FTIR) spectrophotometer using oMNIC software. All spectra were run as $\mathrm{KBr}$ discs. Melting point values were recorded on a Stuart scientific SMP1 melting point apparatus<smiles>[R]c1cc(/C=N/c2ccc3c(C)cc(=O)[nH]c3c2)c([R])c([R])c1[R]</smiles>

Fig. 1. General structure of a quinolin-2(1H)-one derived Schiff base with numbering system used in this report. and are uncorrected. Values were taken up to $300^{\circ} \mathrm{C}$. All NMR spectra were run on a JEOL JNM-LA300 FT-NMR $\left(300 \mathrm{MHz}{ }^{1} \mathrm{H}\right.$ and $75 \mathrm{MHz}{ }^{13} \mathrm{C}$ ) in $d_{6}$-DMSO with ${ }^{1} \mathrm{H}$ NMR spectra recorded in the region of $-5 \mathrm{ppm}$ to $15 \mathrm{ppm}$ from TMS (tetramethylsilane) with a resolution of $0.18 \mathrm{~Hz}$ or $0.0006 \mathrm{ppm}$. All ${ }^{13} \mathrm{C}$ NMR spectra were recorded in the region -33 to $233 \mathrm{ppm}$ from TMS with a resolution of $0.008 \mathrm{ppm}$. Atomic absorption spectroscopy (AAS) measurements were recorded on a Perkin-Elmer 460 AAS instrument (emission wavelength $324.8 \mathrm{~nm}$ ). Solid state magnetic susceptibility measurements were carried out at room temperature using a Johnson Matthey Magnetic Susceptibility Balance with $\left[\mathrm{HgCo}(\mathrm{SCN})_{4}\right]$ being used as a reference standard. Molar conductivity was measured on a Systronic conductivity bridge with a dip-type cell, using $4 \times 10^{-3} \mathrm{M}$ solution of complexes in DMSO. UV-Visible (UV-Vis) spectra of the compounds in DMSO were recorded on a Shimadzu UV-1601 spectrophotometer.

\subsection{Synthesis of 7-amino-4-methylquinolin-2(1H)-one (1)}

7-Amino-4-methylquinolinone (1) was synthesised from 1,3phenylenediamine and ethyl acetoacetate according to a literature procedure [32]. An off-white coloured product 1 was obtained with moderate yield (47\%).

\subsection{Preparation of novel quinolin-2(1H)-one-derived Schiff base derivatives (2-14)}

All of the quinolin-2(1H)-one-derived Schiff base ligands (2-14) were synthesised by an equimolar condensation reaction between substituted aromatic aldehydes and 7-amino-4-methylquinolin$2(1 \mathrm{H})$-one (1) in hot ethanol. A stirred suspension of $\mathbf{1}$ (2 mmol, $0.348 \mathrm{~g})$ and the appropriate aromatic aldehyde $(2 \mathrm{mmol})$ in hot ethanol $(40 \mathrm{~mL})$ was refluxed for $4 \mathrm{~h}$. On cooling to room temperature, coloured precipitates (2-14) formed in good yields $(>70 \%)$, were filtered off and dried in a vacuum oven for two weeks. The general structure of the ligands with the numbering system used in this work is shown in Fig. 1 with detail of the exact structures given in Table 1 . All of the prepared ligands showed poor solubility in polar and non polar solvents at room temperature and most of them were only soluble in hot alcohols, acetonitrile, DMF and DMSO. However, the halogen substituted compounds were soluble only in hot DMSO and DMF. Analytical data for the ligands is given in Tables 3-6.

Table 1

Ligand names, their relative substituents and positions of R groups for compounds (2-14).

\begin{tabular}{|c|c|c|c|c|}
\hline \multirow[t]{2}{*}{ Compound Name and Number } & \multicolumn{4}{|c|}{ R groups } \\
\hline & $\mathrm{R}_{1}$ & $\mathrm{R}_{2}$ & $\mathrm{R}_{3}$ & $\mathrm{R}_{4}$ \\
\hline (7E)-7-(2,4dihydroxybenzylideneamino)-4-methylquinolin-2(1H)-one (2) & $\mathrm{OH}$ & $\mathrm{H}$ & $\mathrm{OH}$ & $\mathrm{H}$ \\
\hline (7E)-7-(2-hydroxybenzylideneamino)-4-methylquinolin-2(1H)-one (3) & $\mathrm{OH}$ & $\mathrm{H}$ & $\mathrm{H}$ & $\mathrm{H}$ \\
\hline (7E)-7-(2,3-dihydroxybenzylideneamino)-4-methylquinolin-2(1H)-one (4) & $\mathrm{OH}$ & $\mathrm{OH}$ & $\mathrm{H}$ & $\mathrm{H}$ \\
\hline (7E)-7-(3-ethoxy-2-hydroxybenzylideneamino)-4-methylquinolin-2(1H)-one (5) & $\mathrm{OH}$ & $\mathrm{OC}_{2} \mathrm{H}_{5}$ & $\mathrm{H}$ & $\mathrm{H}$ \\
\hline (7E)-7-(2-hydroxy-3-methoxybenzylideneamino)-4 methylquinolin-2(1H)-one $(6)$ & $\mathrm{OH}$ & $\mathrm{OCH}_{3}$ & $\mathrm{H}$ & $\mathrm{H}$ \\
\hline (7E)-7-(2-hydroxy-4-methoxybenzylideneamino)-4-methylquinolin-2(1H)-one (7) & $\mathrm{OH}$ & $\mathrm{H}$ & $\mathrm{OCH}_{3}$ & $\mathrm{H}$ \\
\hline (7E)-7-(3,5-di-tert-butyl-2-hydroxybenzylideneamino)-4-methylquinolin-2(1H)-one (8) & $\mathrm{OH}$ & $t \mathrm{Bu}$ & $\mathrm{H}$ & $\mathrm{tBu}$ \\
\hline (7E)-7-(5-bromo-2-hydroxybenzylideneamino)-4-methylquinolin-2(1H)-one $(\mathbf{9})$ & $\mathrm{OH}$ & $\mathrm{H}$ & $\mathrm{H}$ & $\mathrm{Br}$ \\
\hline (7E)-7-(5-chloro-2-hydroxybenzylideneamino)-4-methylquinolin-2(1H)-one $(\mathbf{1 0})$ & $\mathrm{OH}$ & $\mathrm{H}$ & $\mathrm{H}$ & $\mathrm{Cl}$ \\
\hline (7E)-7-(5-fluoro-2-hydroxybenzylideneamino)-4-methylquinolin-2(1H)-one (11) & $\mathrm{OH}$ & $\mathrm{H}$ & $\mathrm{H}$ & $\mathrm{F}$ \\
\hline (7E)-7-(2-hydroxy-5-iodobenzylideneamino)-4-methylquinolin-2(1H)-one (12) & $\mathrm{OH}$ & $\mathrm{H}$ & $\mathrm{H}$ & I \\
\hline (7E)-7-(3-hydroxy-4-methoxybenzylideneamino)-4-methylquinolin-2(1H)-one (13) & $\mathrm{H}$ & $\mathrm{OH}$ & $\mathrm{OCH}_{3}$ & $\mathrm{H}$ \\
\hline (7E)-7-(3-hydroxybenzylideneamino)-4-methylquinolin-2(1H)-one (14) & $\mathrm{H}$ & $\mathrm{OH}$ & $\mathrm{H}$ & $\mathrm{H}$ \\
\hline
\end{tabular}


Table 2

Crystal data and structure refinement of complexes $\mathbf{2 a}$ and $\mathbf{8 a}$.

\begin{tabular}{|c|c|c|}
\hline Empirical formula & $\mathrm{C}_{38} \mathrm{H}_{40} \mathrm{CuN}_{4} \mathrm{O}_{9} \mathrm{~S}(\mathbf{2 a})$ & $\mathrm{C}_{52} \mathrm{H}_{66} \mathrm{CuN}_{4} \mathrm{O}_{6}(\mathbf{8 a})$ \\
\hline Formula weight & 792.34 & 906.63 \\
\hline$T(\mathrm{~K})$ & $100(2)$ & $100(2)$ \\
\hline Wavelength $(\AA)$ & 0.71073 & 0.71073 \\
\hline \multicolumn{3}{|l|}{ Unit cell dimensions } \\
\hline$a(\AA)$ & $10.604(9)$ & $10.07(3)$ \\
\hline$b(\AA)$ & $11.637(11)$ & $10.907(2)$ \\
\hline$c(\AA)$ & $15.027(13)$ & $22.926(6)$ \\
\hline$\alpha\left(^{\circ}\right)$ & $101.661(15)$ & $82.078(14)$ \\
\hline$\beta\left(^{\circ}\right)$ & $96.019(15)$ & $78.592(13)$ \\
\hline$\gamma\left({ }^{\circ}\right)$ & $95.741(18)$ & $69.501(12)$ \\
\hline$V\left(\AA^{3}\right)$ & $1792(3)$ & $2306.5(10)$ \\
\hline$Z$ & 2 & 2 \\
\hline$D_{\text {calc }}\left(\mathrm{Mg} / \mathrm{m}^{3}\right)$ & 1.468 & 1.305 \\
\hline $\begin{array}{l}\text { Absorption coefficient } \\
\left(\mathrm{mm}^{-1}\right)\end{array}$ & 0.730 & 0.528 \\
\hline$F\left(\begin{array}{lll}0 & 0 & 0\end{array}\right)$ & 826 & 966 \\
\hline Crystal size $\left(\mathrm{mm}^{3}\right)$ & $0.38 \times 0.36 \times 0.18$ & $0.55 \times 0.30 \times 0.08$ \\
\hline$\theta$ Range for data collection $\left(^{\circ}\right)$ & $2.24-28.00$ & $0.91-21.82$ \\
\hline Index ranges & $\begin{array}{l}-13 \leqslant h \leqslant 13 \\
-15 \leqslant k \leqslant 15 \\
-18 \leqslant l \leqslant 19\end{array}$ & $\begin{array}{l}-9 \leqslant h \leqslant 10 \\
-10 \leqslant k \leqslant 10 \\
0 \leqslant l \leqslant 23\end{array}$ \\
\hline Reflections collected & 28058 & 5389 \\
\hline Independent reflections & 8562 & 5399 \\
\hline Completeness to $\theta$ & at $25.00^{\circ}=99.9 \%$ & at $21.82^{\circ}=76.3 \%$ \\
\hline $\begin{array}{l}\text { Maximum and minimum } \\
\text { transmission }\end{array}$ & 0.8798 and 0.7688 & 0.9590 and 0.7599 \\
\hline Refinement method & $\begin{array}{l}\text { Full-matrix least- } \\
\text { squares on } F^{2}\end{array}$ & $\begin{array}{l}\text { Full-matrix least- } \\
\text { squares on } F^{2}\end{array}$ \\
\hline Data/restraints/parameters & $8562 / 5 / 500$ & $5399 / 313 / 589$ \\
\hline Goodness-of-fit (GOF) on $F^{2}$ & 0.930 & 1.055 \\
\hline Final $R$ indices $[I>2 \sigma(I)]$ & $\begin{array}{l}R_{1}=0.0550 \\
w R_{2}=0.1284\end{array}$ & $\begin{array}{l}R_{1}=0.1162 \\
w R_{2}=0.2853\end{array}$ \\
\hline$R$ indices (all data) & $\begin{array}{l}R_{1}=0.1062 \\
w R_{2}=0.1572\end{array}$ & $\begin{array}{l}R_{1}=0.1910 \\
w R_{2}=0.3243\end{array}$ \\
\hline $\begin{array}{l}\text { Largest difference in peak } \\
\text { and hole }\left(\mathrm{e} \AA^{-3}\right)\end{array}$ & 0.505 and -0.801 & 0.843 and -0.637 \\
\hline
\end{tabular}

2.4. Synthesis of copper(II) complexes of quinolin-2(1H)-one-derived Schiff base ligands (2a-14a)

All of the copper(II) complexes (2a-14a) were synthesised by the following general procedure. Copper(II) acetate $(0.125 \mathrm{mmol}$ $0.0227 \mathrm{~g})$ (2a-4a, 6a-8a and 10a-14a) or copper(II) perchlorate hexahydrate $(2 \mathrm{mmol}, 0.0463 \mathrm{~g})(\mathbf{5 a}$ and $\mathbf{9 a})$ was dissolved in methanol $(30 \mathrm{~mL})$ and added drop-wise with stirring to a hot methanolic solution of the appropriate ligand $(0.25 \mathrm{mmol})$ to a final volume of $60 \mathrm{~mL}$. The resulting mixture was refluxed with stirring for $5 \mathrm{~h}$ until a precipitate formed. Workup afforded coloured complexes $(\mathbf{2 a}-\mathbf{1 4 a})$ in good yields $(>70 \%)$. All of the complexes were re-crystallised from an appropriate solvent system but suitable crystals were isolated only for complexes $\mathbf{2 a}$ and $\mathbf{8 a}$. Crystals
Table 4

Selected IR and UV-Vis data $\left(\mathrm{cm}^{-1}\right)$ for the Schiff base ligands (2-14).

\begin{tabular}{|c|c|c|c|c|c|}
\hline \multirow[t]{2}{*}{ Ligand } & \multicolumn{4}{|c|}{ IR-spectra $\left(v_{\max } / \mathrm{cm}^{-1}\right)$} & \multirow{2}{*}{$\begin{array}{l}\text { UV-Vis } \\
\lambda_{\max }(\mathrm{nm})\end{array}$} \\
\hline & $-\mathrm{C}=\mathrm{O}($ lactam $)$ & $-\mathrm{OH}$ & $-\mathrm{C}-\mathrm{O}$ & $-\mathrm{CH}=\mathrm{N}$ & \\
\hline 2 & 1651 & 3464 & 1230 & 1575 & $280,342,355$ \\
\hline 3 & 1659 & 3457 & 1269 & 1601 & $297,342,356$ \\
\hline 4 & 1650 & 3388 & 1277 & 1604 & $276,342,355$ \\
\hline 5 & 1650 & 3458 & 1250 & 1599 & $297,342,355$ \\
\hline 6 & 1659 & 3457 & 1251 & 1600 & $296,342,355$ \\
\hline 7 & 1673 & 3456 & 1235 & 1603 & $278,341,356$ \\
\hline 8 & 1654 & 3448 & 1247 & 1608 & 290,357 \\
\hline 9 & 1655 & 3439 & 1276 & 1603 & $\mathrm{n} / \mathrm{m}$ \\
\hline 10 & 1659 & 3446 & 1280 & 1605 & $\mathrm{n} / \mathrm{m}$ \\
\hline 11 & 1692 & 3434 & 1285 & 1608 & $296,342,354$ \\
\hline 12 & 1652 & 3432 & 1280 & 1600 & $\mathrm{n} / \mathrm{m}$ \\
\hline 13 & 1660 & 3442 & 1277 & 1600 & 279,356 \\
\hline 14 & 1653 & 3455 & 1250 & 1613 & $267,283,355$ \\
\hline
\end{tabular}

$\mathrm{n} / \mathrm{m}=$ Not measured due to poor solubility.

suitable for X-ray diffraction measurements were obtained by the slow diffusion of diethylether into a solution of the complex in a binary solution of DMSO-MeOH. The analytical data for the complexes is given in Tables 7 and 8 .

\subsection{X-ray crystallography}

X-ray crystallographic studies were carried out at Heriot Watt University, Edinburgh, United Kingdom. A single crystal is coated in Paratone- $\mathrm{N}$ heavy oil then mounted on a Hampton Research Cryoloop and placed in a cold stream of nitrogen gas $(100 \mathrm{~K})$ on a Bruker Nonius X8 Apex2 CCD diffractometer running the APEX2 [33] software suite. Data collection consisted of, on average, 3000 frames from a combination of phi and omega scans. After unit cell determination, the data were integrated using saint then scaled with SADABS (TWINABS for 8a) before Space group determination with XPREP, structure solution with XS and structure refinement with XL. Pictures are prepared using the graphics program XP. The quality of the datasets is checked using the IUCr checkcif facility. The crystal of 8a was not single and very weakly diffracting and the anisotropy of many atoms was restrained with the SHELXL [34] command ISOR. Crystal data and structure refinement of copper(II) complexes $\mathbf{2 a}$ and $\mathbf{8 a}$ are given in Table 3 .

\subsection{Biological testing}

\subsubsection{Anti-Candida susceptibility testing}

The metal-free ligands (2-14) and their $\mathrm{Cu}(\mathrm{II})$ complexes (2a-14a) were tested for their antifungal activity against a clinical isolate of the fungal strain C. albicans (ATCC 10231) and a commercially available antifungal drug, Amphotericin B. The screening was

Table 3

Analytical data for the series of quinolin-2(1H)-one derived Schiff base ligands (2-14).

\begin{tabular}{|c|c|c|c|c|c|c|c|}
\hline \multirow[t]{2}{*}{ Ligand } & \multirow[t]{2}{*}{$\mathrm{Mw}(\mathrm{g} / \mathrm{mol})$} & \multirow[t]{2}{*}{ Yield (\%) } & \multirow[t]{2}{*}{ Empirical formula } & \multicolumn{3}{|c|}{ Found (Calc) (\%) } & \multirow[t]{2}{*}{ M.P. $\left({ }^{\circ} \mathrm{C}\right)$} \\
\hline & & & & C & $\mathrm{H}$ & $\mathrm{N}$ & \\
\hline 2 & 294.30 & 72 & $\mathrm{C}_{17} \mathrm{H}_{14} \mathrm{~N}_{2} \mathrm{O}_{3}$ & $69.07(69.38)$ & $4.78(4.79)$ & $9.36(9.52)$ & $280-282$ \\
\hline 3 & 278.31 & 79 & $\mathrm{C}_{17} \mathrm{H}_{14} \mathrm{~N}_{2} \mathrm{O}_{2}$ & 73.12 (73.37) & $5.03(5.07)$ & $9.96(10.07)$ & $278-280$ \\
\hline 4 & 294.30 & 74 & $\mathrm{C}_{17} \mathrm{H}_{14} \mathrm{~N}_{2} \mathrm{O}_{3}$ & $69.28(69.38)$ & $4.42(4.79)$ & $9.29(9.52)$ & $298-300$ \\
\hline 5 & 322.36 & 75 & $\mathrm{C}_{19} \mathrm{H}_{18} \mathrm{~N}_{2} \mathrm{O}_{3}$ & $70.62(70.79)$ & $5.60(5.63)$ & $8.68(8.69)$ & $268-270$ \\
\hline 6 & 308.33 & 73 & $\mathrm{C}_{18} \mathrm{H}_{16} \mathrm{~N}_{2} \mathrm{O}_{3}$ & 69.9070 .12 & $5.06(5.23)$ & $9.09(9.09)$ & $288-290$ \\
\hline 7 & 308.33 & 70 & $\mathrm{C}_{18} \mathrm{H}_{16} \mathrm{~N}_{2} \mathrm{O}_{3}$ & $69.60(70.12)$ & $5.18(5.23)$ & $9.07(9.09)$ & $286-288$ \\
\hline 8 & 390.52 & 60 & $\mathrm{C}_{25} \mathrm{H}_{30} \mathrm{~N}_{2} \mathrm{O}_{2}$ & 76.66 (76.89) & $7.63(7.74)$ & $7.22(7.17)$ & $298-300$ \\
\hline 9 & 357.20 & 76 & $\mathrm{C}_{17} \mathrm{H}_{13} \mathrm{BrN}_{2} \mathrm{O}_{2}$ & $57.21(57.16)$ & $3.56(3.67)$ & $7.54(7.84)$ & $>300$ \\
\hline 10 & 312.75 & 78 & $\mathrm{C}_{17} \mathrm{H}_{13} \mathrm{ClN}_{2} \mathrm{O}_{2}$ & $65.42(65.29)$ & $4.06(4.19)$ & $8.92(8.96)$ & $>300$ \\
\hline 11 & 296.30 & 65 & $\mathrm{C}_{17} \mathrm{H}_{13} \mathrm{FN}_{2} \mathrm{O}_{2}$ & $68.64(68.91)$ & $4.25(4.42)$ & $9.15(9.45)$ & $>300$ \\
\hline 12 & 404.2 & 68 & $\mathrm{C}_{17} \mathrm{H}_{13} \mathrm{IN}_{2} \mathrm{O}_{2}$ & $50.82(50.51)$ & $3.35(3.24)$ & $6.88(6.93)$ & $>300$ \\
\hline 13 & 308.33 & 62 & $\mathrm{C}_{18} \mathrm{H}_{16} \mathrm{~N}_{2} \mathrm{O}_{3}$ & $70.01(70.12)$ & $5.02(5.23)$ & $9.22(9.09)$ & $>300$ \\
\hline 14 & 278.31 & 61 & $\mathrm{C}_{17} \mathrm{H}_{14} \mathrm{~N}_{2} \mathrm{O}_{2}$ & 72.57 (73.37) & $5.05(5.07)$ & 10.09 (10.07) & $290-292$ \\
\hline
\end{tabular}


Table 5

Characteristic ${ }^{1} \mathrm{H}$ NMR chemical shift values (ppm), peak multiplicity and coupling constant $J(\mathrm{~Hz})$ for the synthesised ligands recorded in $d_{6}$-DMSO.

\begin{tabular}{|c|c|c|c|c|c|c|c|c|}
\hline \multirow[t]{2}{*}{ Ligand } & \multirow{2}{*}{$\frac{\left(1 \mathrm{H}, \mathrm{s}, \mathrm{H}_{3}\right)}{(\text { vinyl })}$} & \multirow{2}{*}{$\begin{array}{l}(1 \mathrm{H}, \mathrm{s}) \\
(\mathrm{CH}=\mathrm{N})\end{array}$} & \multirow{2}{*}{$\frac{(1 \mathrm{H}, \mathrm{s})}{(\mathrm{NH})}$} & \multirow[t]{2}{*}{$\mathrm{R}_{1}(1 \mathrm{H}, \mathrm{s})$} & \multirow[t]{2}{*}{$\mathrm{R}_{2}$} & \multirow[t]{2}{*}{$\mathrm{R}_{3}$} & \multirow[t]{2}{*}{$\mathrm{R}_{4}$} & \multirow[t]{2}{*}{$\mathrm{H}_{19}(1 \mathrm{H})$} \\
\hline & & & & & & & & \\
\hline 2 & 6.35 & 8.83 & 11.60 & $13.28(\mathrm{OH})$ & $6.31,1 \mathrm{H}, \mathrm{d}, J=2.20$ & $10.37,1 \mathrm{H}, \mathrm{s}, \mathrm{OH}$ & $6.44,1 \mathrm{H}, \mathrm{dd}, J=2.20,8.44$ & $7.50, \mathrm{~d}, J=8.62$ \\
\hline 3 & 6.38 & 8.98 & 11.64 & $12.76(\mathrm{OH})$ & $6.93,1 \mathrm{H}, \mathrm{m}$ & $7.03,1 \mathrm{H}, \mathrm{m}$ & $7.42,1 \mathrm{H}, \mathrm{m}$ & $7.70, \mathrm{dd}, J=1.83,8.07$ \\
\hline 4 & 6.38 & 8.98 & 11.64 & $12.80(\mathrm{OH})$ & n/a (br, OH) & $6.96,1 \mathrm{H}, \mathrm{dd}, J=1.50,7.89$ & $6.79,1 \mathrm{H}, \mathrm{pt}, J=7.70,7.89$ & $7.14, \mathrm{dd}, J=1.50,7.0$ \\
\hline 5 & 6.38 & 8.98 & 11.65 & $12.89(\mathrm{OH})$ & $\begin{array}{l}1.36,3 \mathrm{H}, \mathrm{t}, \mathrm{CH}_{3} J=7.04 .05 \\
2 \mathrm{H}, \mathrm{q}, \mathrm{OCH}_{2,} J=7.0\end{array}$ & $7.14,1 \mathrm{H}, \mathrm{dd}, J=1.31,8.07$ & $6.88,1 \mathrm{H}, \mathrm{pt}, J=7.89,7.89$ & $7.26, \mathrm{dd}, J=1.50,7.34$ \\
\hline 6 & 6.36 & 8.98 & 11.63 & $12.82(\mathrm{OH})$ & $3.61,3 \mathrm{H}, \mathrm{s}, \mathrm{OCH}_{3}$ & $7.13,1 \mathrm{H}, \mathrm{dd}, J=1.50,8.80$ & $6.89,1 \mathrm{H}, \mathrm{pt}, J=7.89,8.07$ & $7.24, \mathrm{dd}, J=2.02,8.34$ \\
\hline 7 & 6.36 & 8.90 & 11.62 & $13.41(\mathrm{OH})$ & $6.51,1 \mathrm{H}, \mathrm{d}, J=2.37$ & $4.06,3 \mathrm{H}, \mathrm{s}, \mathrm{OCH}_{3}$ & $6.57,1 \mathrm{H}, \mathrm{dd}, J=2.38,8.80$ & $7.23, \mathrm{~d}, J=8.62$ \\
\hline 8 & 6.36 & 9.03 & 11.66 & $13.79(\mathrm{OH})$ & $1.31,9 \mathrm{H}, \mathrm{s}, \mathrm{t}-\mathrm{Bu}$ & $7.19,1 \mathrm{H}, \mathrm{d}, J=1.70$ & $1.43,9 \mathrm{H}, \mathrm{s}, \mathrm{t}-\mathrm{Bu}$ & $7.44,1 \mathrm{H}, \mathrm{d}, J=1.86$ \\
\hline 9 & 6.36 & 8.95 & 11.63 & $12.61(\mathrm{OH})$ & $6.95,1 \mathrm{H}, \mathrm{d}, J=8.80$ & $7.54,1 \mathrm{H}, \mathrm{dd}, J=2.57,8.80$ & $-\mathrm{Br}-$ & $7.91, \mathrm{~d}, J=2.57$ \\
\hline 10 & 6.38 & 8.95 & 11.65 & $12.63(\mathrm{OH})$ & $6.99,1 \mathrm{H}, \mathrm{d}, J=8.80$ & $7.42,1 \mathrm{H}, \mathrm{dd}, J=2.57,8.89$ & $-\mathrm{Cl}-$ & $7.78, \mathrm{~d}, J=2.75$ \\
\hline 11 & 6.30 & 8.94 & 11.64 & $12.31(\mathrm{OH})$ & $6.96,1 \mathrm{H}, \mathrm{dd}^{\mathrm{a}}$ & $7.25,1 \mathrm{H}, \mathrm{ddd}^{\mathrm{b}}$ & $-\mathrm{F}-$ & $7.53, \mathrm{dd}^{\mathrm{c}}$ \\
\hline 12 & 6.37 & 8.93 & 11.65 & $12.80(\mathrm{OH})$ & $6.83,1 \mathrm{H}, \mathrm{d}, J=8.62$ & $7.69,1 \mathrm{H}, \mathrm{dd}, J=2.38,8.71$ & $-\mathrm{I}-$ & $8.07, \mathrm{~d}, J=2.41$ \\
\hline 13 & 6.35 & 8.46 & 11.59 & $7.05(1 \mathrm{H})$ & $9.40,1 \mathrm{H}, \mathrm{s}, \mathrm{OH}$ & $3.46,3 \mathrm{H}, \mathrm{s}, \mathrm{OCH}_{3}$ & $7.08,1 \mathrm{H}, \mathrm{d}, J=8.62$ & $7.10, \mathrm{~d}, J=8.62$ \\
\hline 14 & 6.35 & 8.55 & 11.62 & $7.07(1 \mathrm{H})$ & $9.75,1 \mathrm{H}, \mathrm{s}, \mathrm{OH}$ & $6.92,1 \mathrm{H}, \mathrm{m}$ & $7.31,1 \mathrm{H}, \mathrm{m}$ & $7.37, \mathrm{~d}, \mathrm{~m}$ \\
\hline
\end{tabular}

a $J_{\mathbf{H}-\mathbf{H}}+{ }^{2} J_{\mathbf{H}-\mathbf{F}}=4.58,9.17$.

b ${ }^{0} J_{\mathbf{H}-\mathbf{H}}+{ }^{m} J_{\mathbf{H}-\mathbf{H}}+{ }^{1} J_{\mathbf{H}-\mathbf{F}}=3.30,8.99,8.44$.

c $J_{\mathbf{H}-\mathbf{H}}+{ }^{1} J_{\mathbf{H}-\mathbf{F}}=12.27$.

Table 6

Characteristic ${ }^{13} \mathrm{C}$ NMR chemical shift values (ppm), for the ligands recorded in $d_{6}$-DMSO.

\begin{tabular}{|c|c|c|c|c|c|c|c|c|c|}
\hline & C-2 & C3 & C-13 & C-15 & C-5 & C-6 & C-8 & C-18 & C-19 \\
\hline & $(\mathrm{C}=\mathbf{0})$ & (vinyl) & $(\mathbf{C H}=\mathrm{N})$ & (C-OH) & & & & & \\
\hline 2 & 161.40 & 120.0 & 163.5 & 163.0 & 126.20 & 114.50 & 108.50 & 106.90 & 134.90 \\
\hline 3 & 160.22 & 108.05 & 164.08 & 161.79 & 126.10 & 119.30 & 114.63 & 124.40 & 132.62 \\
\hline 4 & 161.90 & 108.10 & 164.63 & 149.80 & 126.16 & 118.99 & 114.59 & 120.40 & 122.73 \\
\hline 5 & 161.81 & 108.13 & 164.38 & 149.46 & 126.14 & 117.23 & 114.57 & 120.44 & 123.96 \\
\hline 6 & 161.79 & 108.13 & 164.23 & 149.50 & 126.17 & 115.86 & 114.60 & 120.44 & 123.69 \\
\hline 7 & 162.00 & 119.92 & 163.5 & 163.14 & 125.85 & 114.24 & 107.60 & 106.89 & 134.0 \\
\hline 8 & 161.17 & 107.48 & 165.20 & 148.89 & 127.31 & 119.94 & 114.09 & 140.11 & 125.38 \\
\hline 9 & 161.8 & 96.89 & 162.0 & 159.50 & 125.20 & 110.28 & 107.78 & 119.0 & 130.74 \\
\hline 10 & 161.79 & 96.89 & 162.10 & 159.50 & 125.10 & 110.0 & 108.0 & 118.0 & 131.0 \\
\hline 11 & 161.86 & 96.75 & 162.38 & 153.05 & 126.17 & 110.38 & 108.01 & $156.61^{a}$ & $116.69^{\mathrm{b}}$ \\
\hline 12 & 161.80 & 96.50 & 162.40 & 159.80 & 125.30 & 110.40 & 107.90 & na & 139.50 \\
\hline 13 & 162.0 & 120.92 & 161.30 & $\mathrm{n} / \mathrm{a}$ & 125.85 & 114.24 & 109.60 & 108.89 & 134.0 \\
\hline 14 & 161.50 & 121.92 & 161.50 & $\mathrm{n} / \mathrm{a}$ & 125.55 & 113.24 & 110.60 & 119.10 & 134.0 \\
\hline
\end{tabular}

$\mathrm{n} / \mathrm{a}=$ Not assigned

${ }^{a} \mathrm{~d}, 1 J_{\mathrm{C}-\mathrm{F}}=67.2 \mathrm{~Hz}$

b d, ${ }^{2} J_{\mathrm{C}-\mathrm{F}}=93.6 \mathrm{~Hz}$.

carried out according to the broth microdilution susceptibility protocol method (NCCLS) [35]. Isolates were grown for $24 \mathrm{~h}$ on SDA plates at $37^{\circ} \mathrm{C}$. Cells were suspended in sterile PBS (phosphate buffered saline) to a concentration equal to a 1.0 McFarland standard $\left(1 \times 10^{6}\right)$ and further diluted $(1: 100)$ with minimal media (MM). All compounds were prepared as $1 \%(\mathrm{v} / \mathrm{v})$ solutions in DMSO and each compound was tested in the range of $1-50 \mu \mathrm{g} \mathrm{mL}^{-1}$. Plates were incubated in an Anthos plate reader for $24 \mathrm{~h}$ at $37^{\circ} \mathrm{C}$ with continuous shaking. Each compound was screened in triplicate and three independent experiments were performed. The stock solution of the compounds which were prepared initially at lower concentration $\left(0.001 \mathrm{~g} \mathrm{~mL}^{-1}\right)$ yielded a clear solution. However, some of the compounds did not dissolve well in DMSO and those compounds were tested as suspensions.

\subsubsection{Antibacterial screening method}

Only those complexes (2a-8a) and their respective ligands (2-8) which showed good solubility in DMSO were tested for their antibacterial activity against a Gram-negative bacterium Escherichia coli and a Gram-positive bacterium Staphylococcus aureus (clinically isolated from wound infections) for their antibacterial activity. The screening was carried out according to a literature reported method [24]. All media, buffers, and solutions used during the course of this study were sterilised by autoclaving at $120^{\circ} \mathrm{C}$ for $15 \mathrm{~min}$. Bacterial strains were grown for $24 \mathrm{~h}$ in nutrient broth medium at $30^{\circ} \mathrm{C}$ and $200 \mathrm{rpm}$ in an orbital incubator. The absorbance of the cultures was measured at $600 \mathrm{~nm}$ using a haemocytometer and cultures were diluted to an optical density of 0.1 . The cell suspension $(50 \mu \mathrm{L})$ was added to the wells of a 96 well plate containing test compounds diluted in nutrient broth medium in serial dilutions from 200 to $0.39 \mu \mathrm{M}$. Test compounds were initially prepared in $100 \%$ DMSO and then diluted with nutrient broth, as above, so that the final concentration of DMSO in the cell suspension was never more than $5 \%(\mathrm{v} / \mathrm{v})$. Plates were incubated at $30^{\circ} \mathrm{C}$ for $24 \mathrm{~h}$. The optical density was measured spectrophotometrically (Dynex Technology) at $540 \mathrm{~nm}$. Each compound was screened in triplicate and three independent experiments were performed. The $\mathrm{MIC}_{50}$ was defined as the concentration of test compound which inhibited 50\% visible growth of the strain in $24 \mathrm{~h}$.

\subsubsection{Assessment of anti-proliferate activity using MTT assay}

Selected compounds (2-8 and $\mathbf{2 a - 8 a )}$ were screened for their in vitro anticancer potential using the human-derived hepatic carcinoma cell line, Hep- $\mathrm{G}_{2}$. Cisplatin, the simple copper(II) salts, the metal-free ligands (2-8) together with the copper(II) complexes (2a-8a) were each dissolved in DMSO, diluted in culture media and used to treat model cells at various drug concentrations for periods of $96 \mathrm{~h}$, prior to MTT assay. The maximum percentage of DMSO present in any well was $0.25 \%(\mathrm{v} / \mathrm{v})$. Cells were seeded in sterile 96 well flat-bottomed plates (Sarstedt) at a density of $2.5 \times 10^{4}$ cells $/ \mathrm{mL}$ and grown in $5 \% \mathrm{CO}_{2}$ at $37^{\circ} \mathrm{C}$. At the end of the required incubation period, a miniaturised viability assay using 
Table 7

Analytical data for the series of copper(II) complexes (2a-14a).

\begin{tabular}{|c|c|c|c|c|c|c|c|c|}
\hline \multirow[t]{2}{*}{ Complex } & \multirow[t]{2}{*}{$\mathrm{Mw}(\mathrm{g} / \mathrm{mol})$} & \multirow{2}{*}{$\begin{array}{l}\mu_{\mathrm{eff}} \\
\text { (B.M.) }\end{array}$} & \multirow{2}{*}{$\begin{array}{l}\text { Yield } \\
(\%)\end{array}$} & \multirow[t]{2}{*}{ Empirical formula } & \multicolumn{4}{|c|}{ Found (Calc) (\%) } \\
\hline & & & & & C & $\mathrm{H}$ & $\mathrm{N}$ & $\mathrm{Cu}$ \\
\hline $2 a$ & 650.14 & 1.69 & 81 & $\mathrm{C}_{34} \mathrm{H}_{26} \mathrm{CuN}_{4} \mathrm{O}_{6}$ & $62.07(62.81)$ & $4.09(4.03)$ & $8.26(8.62)$ & $9.50(9.77)$ \\
\hline $3 a$ & 618.14 & 1.61 & 72 & $\mathrm{C}_{34} \mathrm{H}_{26} \mathrm{CuN}_{4} \mathrm{O}_{4}$ & $65.21(66.06)$ & $4.22(4.24)$ & $8.62(9.06)$ & $9.95(10.28)$ \\
\hline $4 a$ & 650.14 & 1.7 & 88 & $\mathrm{C}_{34} \mathrm{H}_{26} \mathrm{CuN}_{4} \mathrm{O}_{6}$ & $62.20(62.81)$ & $3.99(4.03)$ & $8.41(8.62)$ & $9.92(9.77)$ \\
\hline $5 a$ & 905.15 & 1.77 & 73 & $\mathrm{C}_{38} \mathrm{H}_{34} \mathrm{Cl}_{2} \mathrm{CuN}_{4} \mathrm{O}_{14}$ & $51.05(50.42)$ & $4.13(3.79)$ & $6.20(6.19)$ & $6.74(7.00)$ \\
\hline $6 \mathbf{6 a}$ & 678.19 & 1.73 & 84 & $\mathrm{C}_{36} \mathrm{H}_{30} \mathrm{CuN}_{4} \mathrm{O}_{6}$ & $64.67(63.76)$ & $4.67(4.46)$ & $8.27(8.26)$ & $9.05(9.37)$ \\
\hline $7 a$ & 678.19 & 1.66 & 79 & $\mathrm{C}_{36} \mathrm{H}_{30} \mathrm{CuN}_{4} \mathrm{O}_{6}$ & $63.34(63.76)$ & $4.29(4.46)$ & $8.03(8.26)$ & $9.10(9.37)$ \\
\hline $8 \mathbf{a}$ & 842.57 & 1.7 & 78 & $\mathrm{C}_{50} \mathrm{H}_{58} \mathrm{CuN}_{4} \mathrm{O}_{4}$ & 70.45 (71.27) & $6.67(6.94)$ & $6.39(6.65)$ & $7.10(7.54)$ \\
\hline $9 a$ & 775.93 & 1.66 & 71 & $\mathrm{C}_{34} \mathrm{H}_{24} \mathrm{Br}_{2} \mathrm{CuN}_{4} \mathrm{O}_{4}$ & $51.92(52.63)$ & $3.03(3.12)$ & $6.89(7.22)$ & $7.95(8.19)$ \\
\hline $10 a$ & 687.03 & 1.68 & 73 & $\mathrm{C}_{34} \mathrm{H}_{24} \mathrm{Cl}_{2} \mathrm{CuN}_{4} \mathrm{O}_{4}$ & $59.28(59.44)$ & $3.59(3.52)$ & $7.86(8.15)$ & $8.94(9.25)$ \\
\hline $11 a$ & 654.12 & 1.6 & 76 & $\mathrm{C}_{34} \mathrm{H}_{24} \mathrm{CuF}_{2} \mathrm{~N}_{4} \mathrm{O}_{4}$ & $62.76(62.43)$ & $3.80(3.70)$ & $8.45(8.57)$ & $9.94(9.71)$ \\
\hline $12 a$ & 869.93 & 1.63 & 75 & $\mathrm{C}_{34} \mathrm{H}_{24} \mathrm{CuI}_{2} \mathrm{~N}_{4} \mathrm{O}_{4}$ & $47.10(46.94)$ & $2.93(2.78)$ & $6.16(6.44)$ & $7.55(7.30)$ \\
\hline 13a & 755.92 & 1.69 & 76 & $\mathrm{C}_{34} \mathrm{H}_{22} \mathrm{Cl}_{4} \mathrm{CuN}_{4} \mathrm{O}_{4}$ & $54.12(54.02)$ & $3.06(2.93)$ & $7.59(7.41)$ & $8.14(8.41)$ \\
\hline $14 a$ & 933.72 & 1.62 & 73 & $\mathrm{C}_{34} \mathrm{H}_{22} \mathrm{Br}_{4} \mathrm{CuN}_{4} \mathrm{O}_{4}$ & $43.89(43.73)$ & $2.47(2.37)$ & $5.97(6.00)$ & $6.64(6.81)$ \\
\hline
\end{tabular}

Table 8

Selected IR, UV-Vis and Molar conductivity data $\left(\mathrm{cm}^{-1}\right)$ for the $\mathrm{Cu}(\mathrm{II})$ complexes (2a-14a).

\begin{tabular}{llllll}
\hline \multirow{2}{*}{ Complex } & \multicolumn{2}{l}{ IR-spectra $\left(v_{\max } / \mathrm{cm}^{-1}\right)$} & UV-Vis & Molar conductivity \\
\cline { 2 - 4 } & $-\mathrm{C}=\mathrm{O}$ & $-\mathrm{CH}=\mathrm{N}$ & $-\mathrm{C}-\mathrm{O}$ & $\lambda_{\max }(\mathrm{nm})$ & $\Lambda_{\mathrm{M}}\left[\mathrm{S} \mathrm{mol}^{-1} \mathrm{~cm}^{2}\right]$ \\
\hline $\mathbf{2 a}$ & 1648 & 1585 & 1223 & 307,390 & 1.27 \\
$\mathbf{3 a}$ & 1666 & 1607 & 1247 & $341,355,390$ & 0.51 \\
$\mathbf{4 a}$ & 1664 & 1594 & 1266 & 342,401 & 1.28 \\
$\mathbf{5 a}$ & 1654 & 1604 & 1223 & 299,408 & 14.45 \\
$\mathbf{6 a}$ & 1665 & 1603 & 1245 & $296,340,401$ & 1.6 \\
$\mathbf{7 a}$ & 1644 & 1610 & 1226 & 303,385 & 1.71 \\
$\mathbf{8 a}$ & 1676 & 1612 & 1233 & 301,425 & 0.4 \\
$\mathbf{9 a}$ & 1665 & 1601 & 1264 & $\mathrm{n} / \mathrm{m}$ & $\mathrm{n} / \mathrm{m}$ \\
$\mathbf{1 0 a}$ & 1667 & 1603 & 1264 & $\mathrm{n} / \mathrm{m}$ & $\mathrm{n} / \mathrm{m}$ \\
$\mathbf{1 1 a}$ & 1667 & 1600 & 1268 & $\mathrm{n} / \mathrm{m}$ & $\mathrm{n} / \mathrm{m}$ \\
$\mathbf{1 2 a}$ & 1665 & 1598 & 1261 & $\mathrm{n} / \mathrm{m}$ & $\mathrm{n} / \mathrm{m}$ \\
$\mathbf{1 3 a}$ & 1652 & 1600 & 1259 & $\mathrm{n} / \mathrm{m}$ & $\mathrm{n} / \mathrm{m}$ \\
$\mathbf{1 4 a}$ & 1651 & 1598 & 1260 & $\mathrm{n} / \mathrm{m}$ & $\mathrm{n} / \mathrm{m}$ \\
\hline
\end{tabular}

$\mathrm{n} / \mathrm{m}=$ Not measured due to poor solubility.

3-(4,5-dimethylthiazol-2-yl)-2,5-diphenyl-2H-tetrazolium bromide (MTT) was carried out according to the method described by Mosmann [36]. In metabolically active cells, MTT is reduced by the mitochondrial enzyme succinate dehydrogenase to form insoluble purple formazan crystals that are subsequently solubilised, and the optical density (OD) measured spectrophotometrically. Drug-treated cells were assayed by the addition of $20 \mu \mathrm{L}$ of $5 \mathrm{mg} / \mathrm{mL}$ MTT in $0.1 \mathrm{M}$ phosphate buffer saline (PBS), pH 7.4. Following incubation for $4 \mathrm{~h}$ at $37^{\circ} \mathrm{C}$, the overlying medium was aspirated with a syringe and $100 \mu \mathrm{L}$ of DMSO was added to dissolve the formazan crystals. Plates were agitated at high speed to ensure complete dissolution of crystals and OD was measured at $550 \mathrm{~nm}$ using an Anthos HT-II microtitre plate reader. Viability was expressed as a percentage of solvent-treated control cells. This assay had five replicates and each experiment was carried out on at least three separate occasions. The $\mathrm{IC}_{50}$ was calculated and defined as the drug concentration $(\mu \mathrm{M})$ causing a $50 \%$ reduction in cellular viability.

\section{Results and discussion}

3.1. Synthesis and characterisation of novel quinolin-2(1H)-one derived Schiff base ligands (2-14)

A series of quinolin-2(1H)-one-derived Schiff bases (2-14) were synthesised by an equimolar condensation reaction between substituted aromatic aldehydes and 7-amino-4-methylquinolin$2(1 H)$-one in hot ethanol (Scheme 1). The ligands were characterised by melting point and IR, ${ }^{1} \mathrm{H}$ NMR, ${ }^{13} \mathrm{C}$ NMR, and UV-Vis spectroscopies and CHN analysis and all relevant data is given in Tables 3-6.

The IR spectra of the ligands have been summarised in Table 4. The absence of the aldehydic carbonyl stretching bands and the appearance of the characteristic azomethine $v_{\mathrm{C}=\mathrm{N}}$ bands at $c a$. $1600 \mathrm{~cm}^{-1}$ confirmed the formation of the Schiff bases. The broadness of the $v_{\mathrm{OH}}$ band at $c a .3450 \mathrm{~cm}^{-1}$ may be attributed to the intramolecular hydrogen bond between $\mathrm{CH}=\mathrm{N}_{\text {(imine nitrogen) }}$ and $\mathrm{OH}_{\text {(phenolic) }}[37,38]$. In all of the compounds, the stretching vibration of the lactam carbonyl was observed as a sharp band in the region of $1690-1650 \mathrm{~cm}^{-1}$. Medium intensity bands in the range of $1285-1230 \mathrm{~cm}^{-1}$ were also observed and were attributed to the phenolic $v_{\mathrm{C}-\mathrm{O}}$ vibration [39]. The presence of additional bands between 1577 and $1400 \mathrm{~cm}^{-1}$ are attributed $v_{\mathrm{C}=\mathrm{C}}$ bands of the aromatic rings.

The main ${ }^{1} \mathrm{H}$ NMR signals for each of the ligands are shown in Table 5. Formation of the ligands (2-14) was confirmed by the absence of the $\mathrm{NH}_{2}$ proton signal at $\delta 5.71 \mathrm{ppm}$ assigned to the starting material, 7 -amino-4methyl-quinolin- $2(1 \mathrm{H})$-one and the presence of an azomethine proton signal at 8.46-9.03 ppm. In addition, the disappearance of the aldehydic proton signal from the corresponding starting aldehydes, the appearance of aromatic proton signals in the region 6.51-7.90 ppm and an $\mathrm{OH}$ proton signal between 9.40-13.79 ppm further confirmed the formation of the ligands. The signal corresponding to the hydroxyl group at position $15\left(C_{15}\right)$ was shifted downfield because of intramolecular hydrogen bonding between it and the nitrogen of azomethine $(\mathrm{O}-\mathrm{H} \cdots \mathrm{N}==\mathrm{CH})$ [40]. In ligands $\mathbf{2}, \mathbf{1 3}$ and $\mathbf{1 4}$, where there was no possibility of hydrogen bonding with the azomethine nitrogen, the corresponding signal was shifted upfield. Ligand $\mathbf{2}$ showed two different $\mathrm{OH}$ proton signals at 13.28 (H-bonding) and $10.37 \mathrm{ppm}$ (no H-bonding) for positions 15 and 17, respectively. In the NMR spectrum of 7 , the $\mathrm{OH}$ proton signal was observed downfield at $13.41 \mathrm{ppm}$, whereas for $\mathbf{1 3}$ the $\mathrm{OH}$ signal appeared upfield at $9.40 \mathrm{ppm}$. The upfield shift was attributed to the lack of intramolecular hydrogen bonding with the azomethine nitrogen in the latter compound. The positions of the proton signals compared favourably to literature values of similar Schiff base compounds derived from quinolin-8-amine, (pyridin-2-yl)methanamine and 3,5-di-t-butyl-4 hydroxyaniline reported by Creber et al. [41].

The main ${ }^{13} \mathrm{C}$ NMR signals for the ligands are shown in Table 6. The ${ }^{13} \mathrm{C}$ NMR spectra of the Schiff base were characterised by the presence of characteristic signals at $\mathrm{ca}$. $164-140 \mathrm{ppm}$ of the azomethine carbon $(C=N, C-13)$, lactam carbonyl carbon $(C=O, C-2)$, $\mathrm{C}-\mathrm{OH}(\mathrm{C}-15)$, and $\mathrm{C}-4\left(\mathrm{C}-\mathrm{CH}_{3}\right)$ carbons and the upfield signals corresponding to vinyl (C-3) and the aromatic carbons (C-5, C-6, C-8, $\mathrm{C}-18$, and $\mathrm{C}-19$ ). The spectra were further characterised by the absence of the aldehydic proton signal at ca. $190 \mathrm{ppm}$ from the corre- 


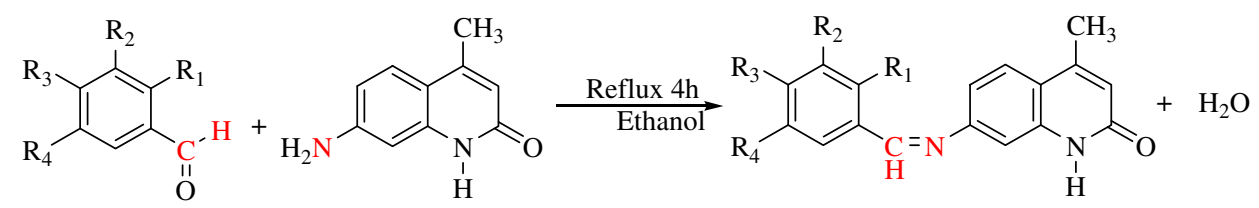

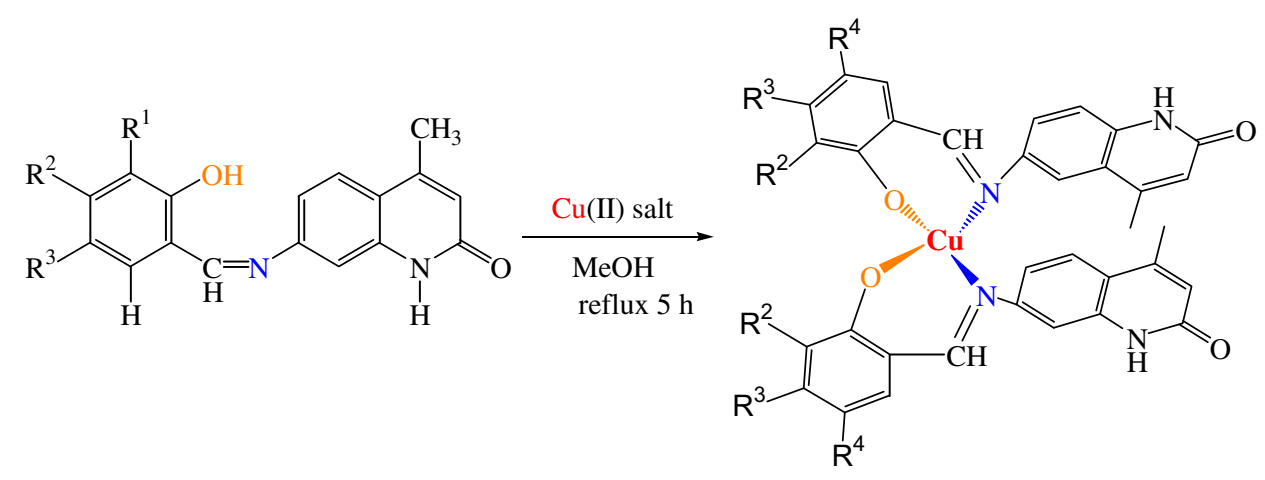

Scheme 1. General reaction scheme for the synthesis of Schiff base ligands and their copper(II) complexes.

sponding aromatic aldehydes (starting material) and the presence of a methyl carbon signal (C-11) at ca.18 ppm. In all of the spectra the azomethine $(\mathrm{C}=\mathrm{N})$ carbon was located at about $163 \mathrm{ppm}$ and the lactam carbonyl was observed at ca.161 ppm. ${ }^{13} \mathrm{C}$ NMR signals at C-5, C-19 and C-15 were affected by the deshielding character of the imine group whereas the ${ }^{13} \mathrm{C}$ NMR signal of the vinyl carbon at C-3 position also appeared downfield because of its proximity to the lactam carbonyl.

\subsection{Synthesis and characterisation of copper(II) complexes (2a-14a)}

Ligands (2-14) were reacted in a 2:1 mole ratio with either copper(II) acetate or copper(II) perchlorate hexahydrate in an appropriate solvent to afford copper(II) complexes (2a-14a) of the corresponding ligands (Scheme 1 ). All of the complexes were characterised by IR, UV-Vis, magnetic moments, molar conductivity, X-ray crystallography where possible, and CHN analyses and the data shown in Tables 7 and 8.

\subsubsection{IR spectra of copper(II) complexes (2a-14a)}

Comparison of the infrared spectral data of complexes (Table 8) and their corresponding ligands (Table 5) confirmed that complexation had occurred as significant shifts in the bands of the azomethine group $v_{\mathrm{CH}=\mathrm{N}}$, lactam $v_{\mathrm{C}=\mathrm{O}}$, phenolic $v_{\mathrm{OH}}$ and $v_{\mathrm{C}-\mathrm{O}}$ groups were observed. The expected mode of interaction between the Schiff base ligand and the copper(II) ion was via coordination of the copper ion to the azomethine nitrogen group and the phenolic oxygen [42]. The strong band at $1604-1575 \mathrm{~cm}^{-1}$ assigned to $v_{(\mathrm{C}=\mathrm{N})}$ in the free ligand was shifted to either lower or higher wavenumber in most of the complexes, indicating the participation of the azomethine nitrogen in the coordination [43]. The medium intensity band at $1285-1230 \mathrm{~cm}^{-1}$ assigned to $v_{(\mathrm{C}-\mathrm{O})}$ in the free ligand was also shifted to lower frequency $\left(1268-1223 \mathrm{~cm}^{-1}\right)$ in the complexes. The change in the intensity of this band confirmed the involvement of the phenolic oxygen in the coordination. Moreover, the appearance of additional weak bands in the region 485-425 and 585$530 \mathrm{~cm}^{-1}$ which were attributed to $v(\mathrm{M}-\mathrm{O})$ and $v(\mathrm{M}-\mathrm{N})$, respectively further confirmed complexation [44].

In the spectra of complex 5a, bands at 1099 and $622 \mathrm{~cm}^{-1}$ were assigned to non-coordinated perchlorates counter anions [45-47] and the presence of these ions was confirmed by elemental analy- sis and molar conductivity results. In contrast to the other complexes isolated, 5a exhibited a sharp band at $3424 \mathrm{~cm}^{-1}$ which was attributed to the phenolic hydroxyl group. Therefore, it was concluded that in complex 5a, the mode of coordination of metal ion to the ligand was via the nitrogen of azomethine and the oxygen atom of the phenolic groups, a coordination mode noted in similar Schiff base complexes of $\mathrm{Ni}(\mathrm{II})$, isolated by Majumder et al. [47].

\subsubsection{Magnetic susceptibility of copper(II) complexes (2a-14a)}

The effective magnetic moment values ( $\mu_{\text {eff }}$ ) of copper(II) complexes were calculated using the formula $\mu_{\text {eff }}=2.84\left(\chi_{\text {corr }} \times T\right)^{1 / 2}$ B.M. The magnetic moment values of the complexes are given in Table 7. The effective magnetic moments obtained for the complexes are in the range of 1.60-1.77 B.M. which is consistent with the presence of one unpaired electron [48]. Generally mononuclear copper(II) complexes (those lacking of $\mathrm{Cu}-\mathrm{Cu}$ interactions) exhibit magnetic moments in the range 1.75-2.20 B.M. corresponding to one unpaired electron $\left(3 \mathrm{~d}^{9} 4 \mathrm{~s}^{0}\right)$, regardless of the stereochemistry $[21,49]$. The observed magnetic moments values are within the expected range for mononuclear copper(II) complexes.

\subsubsection{Electronic spectra of copper(II) complexes (2a-8a)}

The electronic spectral data in DMSO are given for ligands 2-8 and their respective $\mathrm{Cu}(\mathrm{II})$ complexes in Tables 5 and 8 , respectively. The spectra were not recorded for the complexes 9a-14a which had poor solubility in DMSO. The electronic spectral data of 2-8 exhibited two bands in the UV region around 276-305, 340-357 and their complexes (2a-8a) had an additional band at ca. $385-425 \mathrm{~nm}$. The bands at $276-300 \mathrm{~nm}$ were assigned to $\pi \rightarrow \pi^{*}$ intra ligand transitions that were localised on the conjugated system of the aromatic moieties [50-52]. The other absorption bands observed in the region of $340-357 \mathrm{~nm}$ were assigned to the $\pi \rightarrow \pi^{*}$ and $n \rightarrow \pi^{*}$ electronic transitions of the azomethine and the lactam carbonyl groups $[53,54]$. The electronic spectra of the complexes showed an additional broad band tailing into the visible region around 385-425 $\mathrm{nm}$ which was not seen in the free ligands. This band was assigned to the ligand-to-metal charge-transfer transition. The expected characteristic $d-d$ transitions in the visible region for the complexes were not detected even when concentrated solutions were used. It was assumed that the bands were lost or 
hidden under the dominant and overlapping charge-transfer band at $385-425 \mathrm{~nm}$ as has been found for other Schiff base systems [55-57].

\subsubsection{Molar conductivity of copper(II) complexes (2a-8a)}

The molar conductivities of copper(II) complexes (2a-8a) in DMSO solvent $\left(4.0 \times 10^{-3} \mathrm{M}\right)$ are given in Table 8 . Conductance measurements were not carried out for all of the complexes due to their poor solubility. The conductance values measured (in the range $0.40-14.45 \mathrm{~S} \mathrm{~cm}^{2} \mathrm{~mol}^{-1}$ ) were too low to account for any dissociation, hence the complexes were considered nonelectrolytes. 5a had a higher molar conductivity than the other complexes due to the presence of two perchlorates anions in the complex.

\subsubsection{Crystal structure of copper(II) complexes $\mathbf{2 a}$ and $\mathbf{8 a}$}

Crystal data and structure refinement of compounds $\mathbf{2 a}$ and $\mathbf{8 a}$ are given in Table 2 and selected bond lengths and angles are presented in Table 9 . The X-ray crystal structure and the crystal packing diagrams for $\mathbf{2 a}$ are shown in Figs. 2 and 3, respectively. From Fig. 2 it was clearly observed that the Schiff base ligand 2 acted as a monoanionic bidentate $\mathrm{N}$, O-donor chelate and formed a neutral, mononuclear, four coordinated complex [58]. Two $\mathrm{MeOH}$ molecules and one DMSO molecule are also present within the asymmetric unit. The ligand coordinated to the copper(II) ion via the phenolic oxygen (at C15) and the nitrogen of the imine bond as expected $[59,60]$. The copper atom was surrounded by a $\mathrm{N}_{2} \mathrm{O}_{2}$ donor set formed by the phenolic oxygens: $\mathrm{Cu} 1-\mathrm{O} 20=1.915(3)$ and $\mathrm{Cu} 1-$ $01=1.916(3) \AA$ and the nitrogen of azomethines from two ligand molecules: Cu1-N28 = 1.964(3), Cu1-N9 = 1.975(3) Å. In all of the four copper-ligand bond distances, the bond distances between $\mathrm{Cu}-\mathrm{N}$ are significantly longer than those of $\mathrm{Cu}-\mathrm{O}$ bonds. Elongated $\mathrm{Cu}-\mathrm{N}$ bonds have also been observed for other Schiff base complexes [61]. The largest distortion from regular square planar geometry is shown in the trans $\mathrm{O}-\mathrm{Cu}-\mathrm{N}$ angles $\left[145.69(11)^{\circ}\right.$ and $\left.140.36(11)^{\circ}\right]$. In similar structures the geometry around the metal centre was also considered as distorted square planar coordination with significant tetrahedral distortion [62-68]. Due to the coordination of the metal ion to the phenolic oxygen, reduced bond distances were observed for coordinated $\mathrm{C}-\mathrm{O}[01-\mathrm{C} 2=1.329(4) \AA]$ compared to non-coordinated $\mathrm{C}-\mathrm{O}[\mathrm{O} 4-\mathrm{C} 4=1.359(4) \AA]$ bond lengths.

Table 9

Selected bond lengths $(\AA)$ lengths and angles $\left(^{\circ}\right)$ for complexes $\mathbf{2 a}$ and $\mathbf{8 a}$.

\begin{tabular}{lllr}
\hline (2a) Bond lengths $(\AA)$ & \multicolumn{3}{l}{$(\mathbf{2 a})$ Bond angles $\left(^{\circ}\right)$} \\
\hline $\mathrm{Cu}(1)-\mathrm{O}(20)$ & $1.915(3)$ & $\mathrm{O}(20)-\mathrm{Cu}(1)-\mathrm{O}(1)$ & $95.10(11)$ \\
$\mathrm{Cu}(1)-\mathrm{O}(1)$ & $1.916(3)$ & $\mathrm{O}(20)-\mathrm{Cu}(1)-\mathrm{N}(28)$ & $95.20(12)$ \\
$\mathrm{Cu}(1)-\mathrm{N}(28)$ & $1.964(3)$ & $\mathrm{O}(1)-\mathrm{Cu}(1)-\mathrm{N}(9)$ & $94.61(11)$ \\
$\mathrm{Cu}(1)-\mathrm{N}(9)$ & $1.975(3)$ & $\mathrm{N}(28)-\mathrm{Cu}(1)-\mathrm{N}(9)$ & $98.01(12)$ \\
$\mathrm{O}(1)-\mathrm{C}(2)$ & $1.329(4)$ & $\mathrm{O}(1)-\mathrm{Cu}(1)-\mathrm{N}(28)$ & $145.69(11)$ \\
$\mathrm{C}(4)-\mathrm{O}(4)$ & $1.359(4)$ & $\mathrm{O}(20)-\mathrm{Cu}(1)-\mathrm{N}(9)$ & $140.36(11)$ \\
$\mathrm{O}(20)-\mathrm{C}(21)$ & $1.310(4)$ & $\mathrm{C}(2)-\mathrm{O}(1)-\mathrm{Cu}(1)$ & $127.8(2)$ \\
$\mathrm{C}(27)-\mathrm{N}(28)$ & $1.310(4)$ & $\mathrm{C}(8)-\mathrm{N}(9)-\mathrm{Cu}(1)$ & $123.0(2)$ \\
$\mathrm{N}(28)-\mathrm{C}(29)$ & $1.441(4)$ & $\mathrm{C}(10)-\mathrm{N}(9)-\mathrm{Cu}(1)$ & $119.0(2)$ \\
$\mathrm{O}(20)-\mathrm{C}(21)$ & $1.310(4)$ & $\mathrm{C}(11)-\mathrm{C}(10)-\mathrm{N}(9)$ & $121.9(3)$ \\
& & $\mathrm{C}(21)-\mathrm{O}(20)-\mathrm{Cu}(1)$ & $125.9(2)$ \\
$(8 \mathbf{a}) \mathrm{Bond}$ lengths $(\AA)$ & & $(\mathbf{8 a}) \mathrm{Bond}$ angles $\left(^{\circ}\right)$ & \\
\hline $\mathrm{Cu}(1)-\mathrm{O}(1)$ & $1.845(9)$ & $\mathrm{O}(1)-\mathrm{Cu}(1)-\mathrm{O}(29)$ & $101.5(4)$ \\
$\mathrm{Cu}(1)-\mathrm{O}(29)$ & $1.866(10)$ & $\mathrm{O}(1)-\mathrm{Cu}(1)-\mathrm{N}(9)$ & $93.1(4)$ \\
$\mathrm{Cu}(1)-\mathrm{N}(9)$ & $1.920(11)$ & $\mathrm{O}(29)-\mathrm{Cu}(1)-\mathrm{N}(9)$ & $139.5(5)$ \\
$\mathrm{Cu}(1)-\mathrm{N}(37)$ & $1.934(12)$ & $\mathrm{O}(1)-\mathrm{Cu}(1)-\mathrm{N}(37)$ & $133.2(5)$ \\
$\mathrm{O}(1)-\mathrm{C}(2)$ & $1.322(16)$ & $\mathrm{O}(29)-\mathrm{Cu}(1)-\mathrm{N}(37)$ & $92.9(5)$ \\
$\mathrm{C}(8)-\mathrm{N}(9)$ & $1.284(16)$ & $\mathrm{N}(9)-\mathrm{Cu}(1)-\mathrm{N}(37)$ & $104.3(5)$ \\
$\mathrm{N}(9)-\mathrm{C}(10)$ & $1.392(17)$ & $\mathrm{O}(1)-\mathrm{Cu}(1)$ & $128.2(8)$ \\
& & $\mathrm{C}(8)-\mathrm{N}(9)-\mathrm{Cu}(1)$ & $125.8(9)$ \\
& & $\mathrm{C}(30)-\mathrm{O}(29)-\mathrm{Cu}(1)$ & $128.7(9)$ \\
& & $\mathrm{C}(36)-\mathrm{N}(37)-\mathrm{Cu}(1)$ & $126.6(10)$ \\
\hline
\end{tabular}

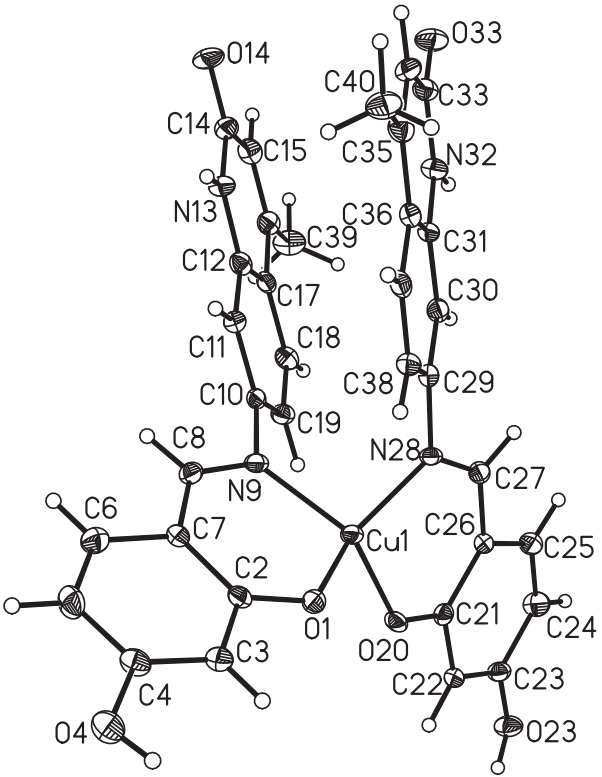

Fig. 2. Crystal structure of complex 2a. Displacement ellipsoids drawn at the 50\% probability level.

The two quinolinone moieties were arranged in cis rather than trans positions [69]. Hence, there are potential $\pi-\pi$ stacking interactions where the centroid-centroid distance is 3.485(4) $\AA$ (though the slippage is $0.884 \AA$ ) between the ring C31-C36 and its symmetry equivalent generated by the symmetry operation $-\mathrm{x},-\mathrm{y}, 1-\mathrm{z}$ [70]. This arrangement is complemented by centrosymmetric $\mathrm{NH}$... O hydrogen bonded ring with a graph set notation of R4,4(8) [71] which holds two adjacent complexes in a dimeric arrangement. This hydrogen bonding motif is undertaken by only one of the two quinolinone groups. The $\mathrm{NH}$ in the other quinolinone group makes a single $\mathrm{NH} \cdots \mathrm{O}$ hydrogen bond with the DMSO solvent whilst the lactam carbonyl (014) makes an O ...HO hydrogen bond with the uncoordinated [72] phenolic oxygen (04). Intermolecular hydrogen bonds with solvent molecules extend the hydrogen bonding network into three dimensions, assisting in stabilising the crystal structure [73]. These hydrogen bonds are shown in Table 10.

The individual metal complexes are stabilised by the formation of two adjacent six-membered chelate rings [74]. Both rings are almost planar with Cu1 lying 0.095 A off the plane defined by N9, C2, C7, C8, 01 but lying $0.278 \AA$ from the plane defined by O20, C21, $\mathrm{C} 26, \mathrm{C} 27, \mathrm{~N} 28$. The benzyl and quinolinone sections are not co planar, with the angle between the planes defined by the atoms forming the aromatic rings (benzene ring $\mathrm{C} 2$ to $\mathrm{C} 7$ ) and (quinoline $\mathrm{C} 10$ to $C 19$ ) being $52.9(2)^{\circ}$. In the other, second Schiff base ligand, the equivalent angle is $30.3(2)^{\circ}$ which is significantly less twisted. This second ligand also has the larger deviation of $\mathrm{Cu} 1$ from the plane defined by the chelating ring. The bond lengths and bond angles are almost identical in both chelate rings.

The crystal structure 8a (Figs. 4 and 5) also showed that the mode of coordination of ligand $\mathbf{8}$ to copper(II) ion was via the phenolic oxygen (at $\mathrm{C} 15$ ) and the nitrogen of the imine bond as observed for the complex 2a. Selected bond lengths and bond angles are presented in Table 11. The two quinolinone moieties were again arranged in cis rather than trans positions and there were two $\mathrm{MeOH}$ solvent molecules also present within the asymmetric unit. The copper atom was surrounded by a $\mathrm{N}_{2} \mathrm{O}_{2}$ donor set formed by the phenolic oxygens $\mathrm{Cu} 1-\mathrm{O} 1=1.845(9)$ and $\mathrm{Cu} 1-$ $\mathrm{O} 29=1.866(3) \AA$ and the nitrogen of azomethines from two molecules of ligand Cu1-N9 = 1.920(3) and Cu1-N37 = 1.934(3) Å. In all 


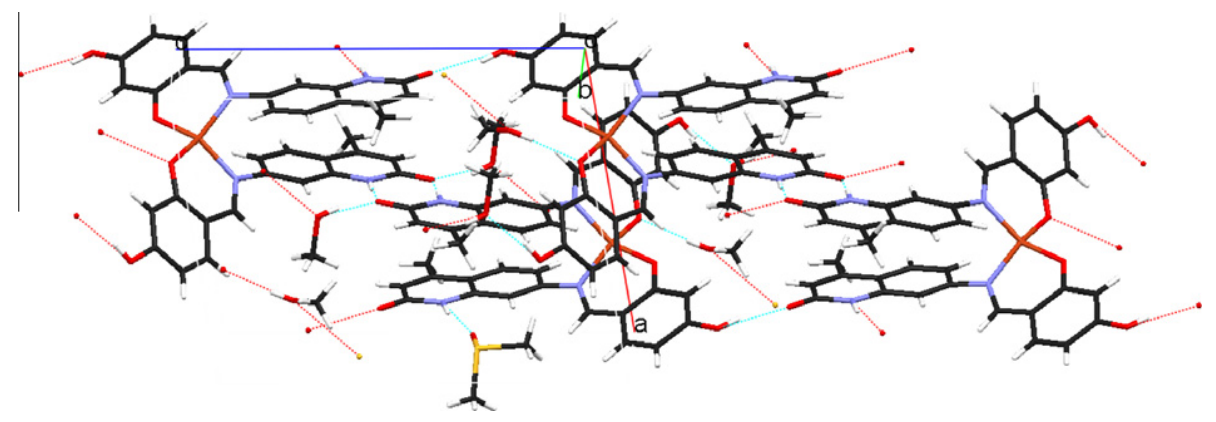

Fig. 3. Crystal packing structure for complex $\mathbf{2 a}$.

of the four copper-ligand bond distances, the bond distances between $\mathrm{Cu}$ and $\mathrm{N}$ are significantly larger than those of $\mathrm{Cu}-\mathrm{O}$ bonds. However, these bond distances are slightly smaller than the bond distances observed for complex 2a but can be regarded as normal compared with the distances found in similar Schiff base compounds [69]. 8a exhibited similar trans $\left[139.5(5)^{\circ}, 133.2(5)^{\circ}\right]$ and bite $\left[93.1(4)^{\circ}, 92.9(5)^{\circ}\right]$ angles around the metal centre. The geometry around the metal centre was also considered as distorted square planar with significant tetrahedral distortion arrangement as observed for complex 2a. The same centrosymmetric N-H ‥ dimeric hydrogen bonding arrangement is present in $\mathbf{8 a}$ as in $\mathbf{2 a}$, but in $\mathbf{8 a}$, both quinolinone groups have the R4,4(8) network. However the phenol ring substituents are all hydrocarbons so the network exists as a stack propagated along the $a$ axis as opposed to the three-dimensional network found in $\mathbf{2 a}$. The quinolinone rings appear to stack in a way to allow $\pi-\pi$ interactions but the closest centroid-centroid distance is 3.719(9) A. The hydrogen bonds are listed in Table 11. The solvent molecules $(\mathrm{MeOH})$, present in the crystal structure do not extend the hydrogen bonding network beyond the stack being linked to one uncoordinated lactam carbonyl (017 or 045) each.

\section{Biological studies}

4.1. Antimicrobial activity of Schiff base ligands and their copper(II) complexes

The anti-Candida activity of the ligands (2-14), their copper (II) complexes (2a-14a) and the metal salts used in the synthesis of the complexes is given in Table 12 expressed as $\mathrm{MIC}_{50}$ values (a minimal concentration required to inhibit a $50 \%$ of cells growth). No inhibition of growth was found for the free copper(II) salts. Most of the quinolinone ligands and their copper(II) complexes displayed considerable activity against $C$. albicans, comparable to that of a commercially available antifungal drug, Amphotericin B. If one considers the activity of the ligands first, the most active ligands are those with either two hydroxyl groups or one hydroxyl group and an alkoxy group on the phenyl ring (4, 5, 6, and 7). However,

Table 10

Hydrogen bond parameters for $\mathbf{2 a}$.

\begin{tabular}{lllll}
\hline $\mathrm{D}-\mathrm{H} \cdots \mathrm{A}$ & $\mathrm{d}(\mathrm{D}-\mathrm{H})$ & $\mathrm{d}(\mathrm{H} \cdots \mathrm{A})$ & $\mathrm{d}(\mathrm{D} \cdots \mathrm{A})$ & $\angle(\mathrm{DHA})$ \\
\hline $\mathrm{O}(4)-\mathrm{H}(4) \cdots \mathrm{O}(1 \mathrm{~S}) \# 1$ & 0.84 & 1.83 & $2.659(4)$ & 168.9 \\
$\mathrm{~N}(13)-\mathrm{H}(13) \cdots \mathrm{O}(14) \# 2$ & $0.81(4)$ & $1.98(4)$ & $2.793(5)$ & $172(4)$ \\
$\mathrm{O}(23)-\mathrm{H}(23) \cdots \mathrm{O}(33) \# 3$ & 0.84 & 1.96 & $2.678(4)$ & 142.5 \\
$\mathrm{~N}(32)-\mathrm{H}(32) \cdots \mathrm{O}(3 \mathrm{~S}) \# 4$ & $0.93(4)$ & $1.96(4)$ & $2.867(4)$ & $163(4)$ \\
$\mathrm{O}(1 \mathrm{~S})-\mathrm{H}(1 \mathrm{~S}) \cdots \mathrm{O}(14)$ & 0.84 & 1.88 & $2.698(4)$ & 165.9 \\
$\mathrm{O}(2 \mathrm{~S})-\mathrm{H}(2 \mathrm{~S}) \cdots \mathrm{O}(1) \# 5$ & 0.84 & 1.97 & $2.809(4)$ & 175.1 \\
\hline
\end{tabular}

Note: Symmetry transformations used to generate equivalent atoms: (i) $x, y-1$, $z-1$; (ii) $-x+1,-y,-z+1$; (iii) $x, y, z-1$; (iv) $x+1, y, z$; (v) $x, y+1, z+1$.

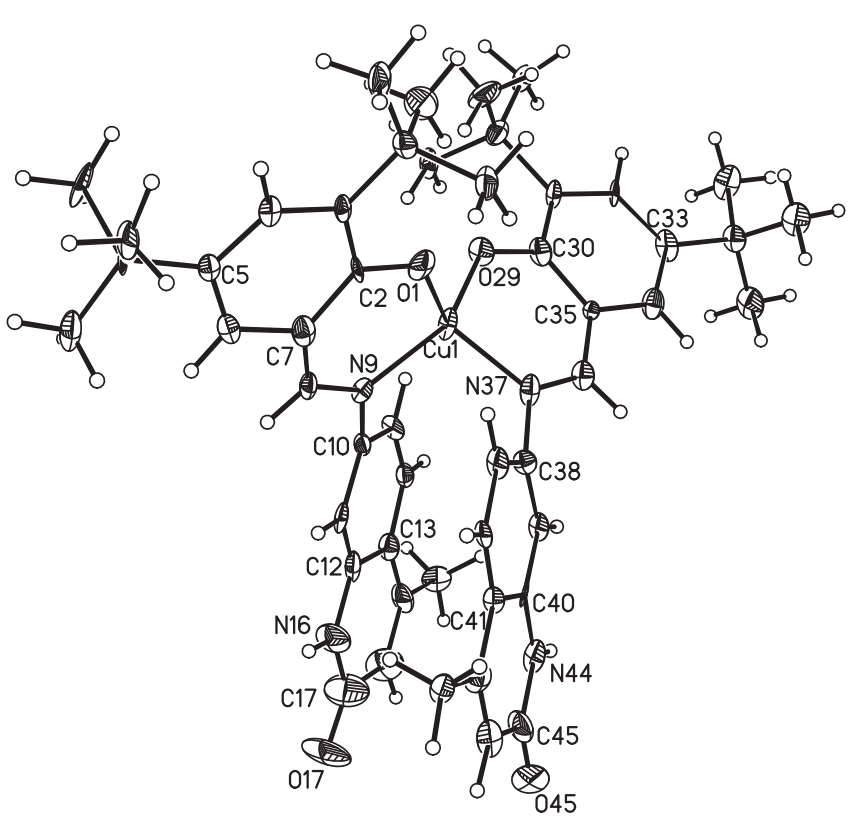

Fig. 4. Crystal structure of complex 8a. Displacement ellipsoids drawn at the $40 \%$ probability level.

ligand 2 also has 2 hydroxy groups on the aromatic ring but they are $m$ - rather than $o$-dihydroxy. Of the halogen substituted compounds, the activity of the fluoro-derivative was considerable better than that of the iodo-, bromo- or chloro- analogues. A similar, trend was also reported by Kalkhambkar et al. in their antimicrobial activity of a series of halogenated $(\mathrm{F}, \mathrm{Cl}$ and $\mathrm{Br}$ ) quinolin$2(1 \mathrm{H})$-one and coumarin derivatives where the fluoro-derivatives showed excellent activity compared to the corresponding chloro and bromo derivatives. Despite these findings, it is difficult to identify a structure activity relationship (SAR) with respect to electronic and steric properties of a compound. It was found that substitution of a methoxy group (15) in place of ethoxy group (14) had little effect on activity indicating that the bulky nature of the substituent has no effect on the activity of a compound at this position. It may prove difficult to identify a real SAR when the target of the ligands remained unidentified to date.

In general it can be seen that coordination to copper(II) resulted in improved activity and all of the complexes, with the exception of the fluoro-derivative, which exhibited greater activity $\left(\mathrm{IC}_{50}=\sim 4-\right.$ $51 \mu \mathrm{M})$ than their corresponding free ligands $\left(\mathrm{IC}_{50}=\sim 10-80 \mu \mathrm{M}\right)$. The decreased activity of the complex 11a compared to that of ligand $\mathbf{1 1}$ could be attributed to its poor solubility but this should also apply to complexes $\mathbf{9 a - 1 4 a}$, as all of these complexes were considerably less soluble than their respective free ligands. We propose that complexation to the copper(II) ion may block the tar- 


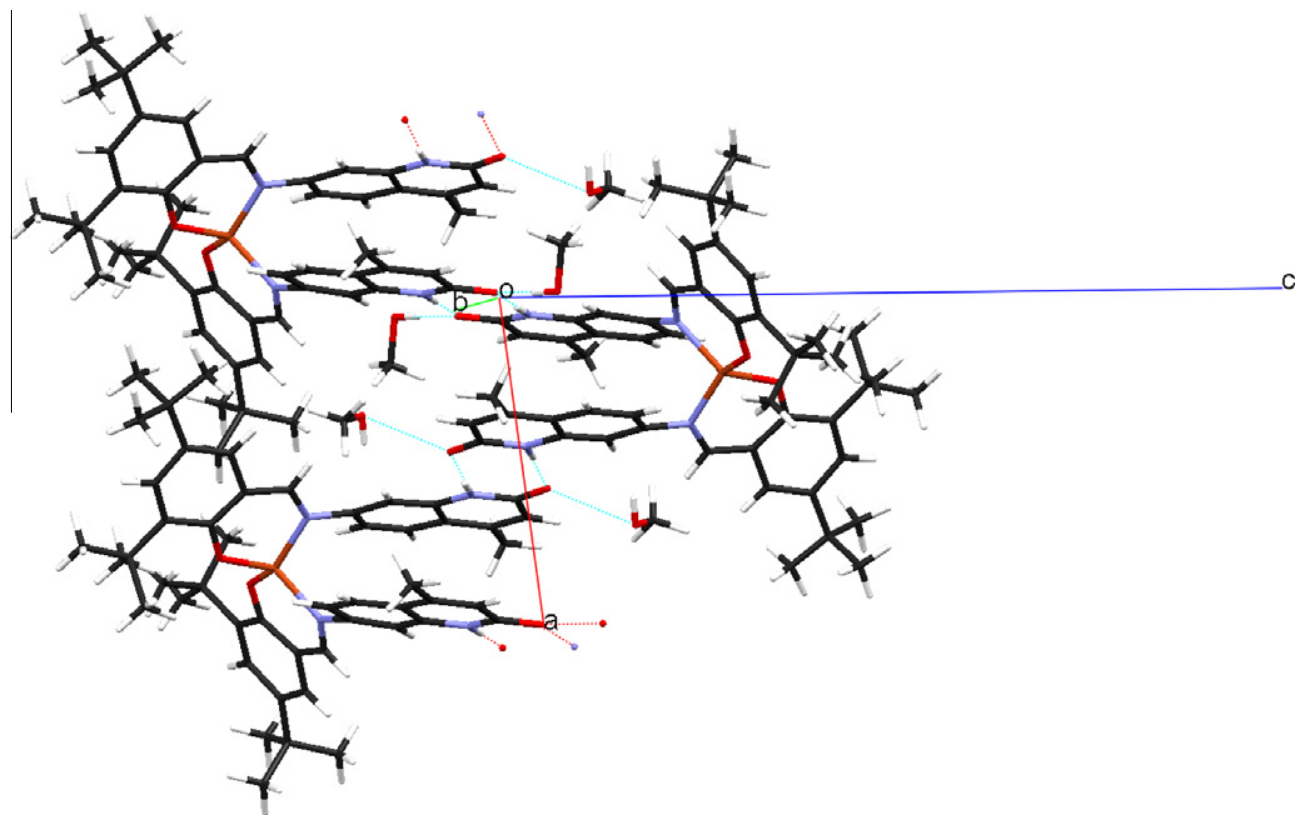

Fig. 5. Crystal packing structure for complex $\mathbf{8 a}$.

Table 11

Hydrogen bond parameters for $\mathbf{8 a}$.

\begin{tabular}{lllll}
\hline $\mathrm{D}-\mathrm{H} \cdots \mathrm{A}$ & $\mathrm{d}(\mathrm{D}-\mathrm{H})$ & $\mathrm{d}(\mathrm{H} \cdots \mathrm{A})$ & $\mathrm{d}(\mathrm{D} \cdots \mathrm{A})$ & $\angle(\mathrm{DHA})$ \\
\hline $\mathrm{N}(44)-\mathrm{H}(44) \cdots \mathrm{N}(44) \# 1$ & 0.88 & 2.81 & $3.46(2)$ & 132.0 \\
$\mathrm{~N}(44)-\mathrm{H}(44) \cdots \mathrm{O}(45) \# 1$ & 0.88 & 1.87 & $2.745(16)$ & 176.3 \\
$\mathrm{~N}(16)-\mathrm{H}(16) \cdots \mathrm{O}(17) \# 2$ & 0.88 & 1.87 & $2.743(18)$ & 171.8 \\
$\mathrm{O}(58)-\mathrm{H}(58) \cdots \mathrm{O}(17) \# 3$ & 0.84 & 2.54 & $2.720(17)$ & 93.4 \\
$\mathrm{O}(60)-\mathrm{H}(60) \cdots \mathrm{O}(45) \# 3$ & $0.889(19)$ & $1.84(3)$ & $2.722(16)$ & $169(14)$ \\
\hline
\end{tabular}

Note: Symmetry transformations used to generate equivalent atoms: $-x,-y+1,-z$ $x, y, z+1$.

get site of the ligand. The trend in activity of the complexes mirrors those seen with the ligands, with complexes $\mathbf{4 a - 7 a}$ showing good activity. In fact all of the complexes have good activity except the fluoro- and iodo-derivatives, but as many of the complexes had to be tested as suspensions, it is difficult to assign clearly defined SAR's.

The in vitro antibacterial activity of the most soluble Schiff base ligands (2-8), their metal(II) complexes (2a-8a), the starting com-

Table 12

Anti Candida activity of ligands and their copper(II) complexes expressed as $\mathrm{MIC}_{50}$.

\begin{tabular}{llll}
\hline Ligand & $\mathrm{MIC}_{50}(\mu \mathrm{M})$ & Complex & $\mathrm{MIC}_{50}(\mu \mathrm{M})$ \\
\hline $\mathbf{1}$ & 36.9 & - & - \\
$\mathbf{2}$ & 90.4 & $\mathbf{2 a}$ & 8.2 \\
$\mathbf{3}$ & 95.6 & $\mathbf{3 a}$ & 11.8 \\
$\mathbf{4}$ & 10.5 & $\mathbf{4 a}$ & 4.5 \\
$\mathbf{5}$ & 17.1 & $\mathbf{5 a}$ & 4.3 \\
$\mathbf{6}$ & 17.2 & $\mathbf{6 a}$ & 4.3 \\
$\mathbf{7}$ & 17.6 & $\mathbf{7 a}$ & 7.4 \\
$\mathbf{8}$ & 26.7 & $\mathbf{8 a}$ & 19.8 \\
$\mathbf{9}$ & 29.1 & $\mathbf{9 a}$ & 8.9 \\
$\mathbf{1 0}$ & 98.4 & $\mathbf{1 0 a}$ & 8.6 \\
$\mathbf{1 1}$ & 18.2 & $\mathbf{1 1 a}$ & 57.5 \\
$\mathbf{1 2}$ & 80.1 & $12 \mathrm{a}$ & 51.5 \\
$\mathbf{1 3}$ & $>100$ & - & - \\
$\mathbf{1 4}$ & $>100$ & - & - \\
Cu(II) salts & $>100$ & - & - \\
Amphotericin B & 0.61 & - & - \\
\hline
\end{tabular}

pound $\mathbf{1}$ and the metal salts used in the synthesis of the complexes were also determined. Surprisingly, none of the ligands or their copper(II) complexes showed bacterial growth inhibition at maximum concentrations of $200 \mu \mathrm{M}$ (data not shown). Inactivity against both Gram-negative and Gram-positive species recorded for the ligands and complexes together with their significant anti-Candida activity suggested that they are highly selectivity against fungal pathogens. The selectivity might be result from the well established structural differences between fungal and bacterial cells [75], although the exact reasons remain as yet unclear.

\subsection{Anticancer activity of Schiff base ligands and their copper(II) complexes}

Selected compounds (2-8 and $\mathbf{2 a - 8 a )}$ were also screened for their in vitro anticancer potential using the human hepatic carcinoma cell line, Hep- $\mathrm{G}_{2}$. The anticancer activity of Schiff base ligands (2-8) and their $\mathrm{Cu}(\mathrm{II})$ complexes (2a-8a) were expressed as $\mathrm{IC}_{50} \pm$ S.E.M values as shown in Table 13 . With the exception of compounds $\mathbf{2}$ and $\mathbf{8}$, all of the tested metal-free ligands showed moderate cytotoxicity $\left(\mathrm{IC}_{50}=\sim 175-1000 \mu \mathrm{M}\right)$. However, 2 and $\mathbf{8}$ were capable of killing cancer cells at low concentration $\left(\mathrm{IC}_{50}=39.67 \pm 5.24\right.$ and $\left.44.33 \pm 4.70 \mu \mathrm{M}\right)$. The solubility of all of these ligands in complete culture media was very good and it is very difficult to see a correlation between compounds $\mathbf{2}$ and $\mathbf{8}$ in

Table 13

Anticancer activity of ligands and their copper(II) complexes expressed $\mathrm{IC}_{50}(\mu \mathrm{M})$ values as measured using Hep-G2 cell line following $96 \mathrm{~h}$ exposure.

\begin{tabular}{llll}
\hline Ligand & $\mathrm{IC}_{50}(\mu \mathrm{M})$ & Complex & $\mathrm{IC}_{50}(\mu \mathrm{M})$ \\
\hline $\mathbf{1}$ & $>1000$ & - & - \\
$\mathbf{2}$ & $39.67 \pm 5.24$ & $\mathbf{2 a}$ & $47.00 \pm 9.54$ \\
$\mathbf{3}$ & $411.66 \pm 7.26$ & $\mathbf{3 a}$ & $74.83 \pm 8.91$ \\
$\mathbf{4}$ & $>1000$ & $\mathbf{4 a}$ & $129 \pm 16.26$ \\
$\mathbf{5}$ & $398.33 \pm 33.21$ & $\mathbf{5 a}$ & $17.90 \pm 3.75$ \\
$\mathbf{6}$ & $175.67 \pm 12.20$ & $\mathbf{6 a}$ & $105.33 \pm 25.41$ \\
$\mathbf{7}$ & $521.67 \pm 41.26$ & $\mathbf{7 a}$ & $53.17 \pm 1.59$ \\
$\mathbf{8}$ & $44.33 \pm 4.70$ & $\mathbf{8 a}$ & $39.67 \pm 10.18$ \\
Cisplatin & $15.00 \pm 2.65$ & - & - \\
\hline
\end{tabular}


terms of a SAR. Given the presence of two t-butyl groups on the aromatic ring (8), it is realistic to expect that it is quite lipophilic, whilst the m-dihydroxy substituted ligand (2) should be quite hydrophilic.

It is interesting to note that, most of the complexes (except 2a) were found to be more active ( $\mathrm{IC}_{50}=\sim 17-129 \mu \mathrm{M}$ ) than the corresponding free ligands. The overall order of the observed cytotoxicity of the complexes was $5 a>8 a>2 a>7 a>3 a \gg 6 a>4 a$, with $5 a$ appearing to be the most potent and $\mathbf{4 a}$, the least potent. More importantly, the cytotoxicity $\left(\mathrm{IC}_{50}=17.90 \pm 3.75 \mu \mathrm{M}\right)$ of complex 5a was comparable to that of the commercially available drug cisplatin $\left(\mathrm{IC}_{50}=15.00 \pm 2.65 \mu \mathrm{M}\right)$.

\section{Conclusion}

In conclusion, a number of the novel ligands and complexes investigated here had a potent antifungal effect being capable of inhibiting growth of $C$. albicans by $50 \%$ even at a concentration of $\sim 4 \mu \mathrm{M}(\mathbf{4 a}, 5 \mathbf{a}$ and $\mathbf{6 a})$. Hence, it could be concluded that although these compounds did not show any significant antibacterial properties, they may well function as effective antifungal agents. Moreover, the copper(II) complex 5a was found to exhibit potent cytotoxicity, suggesting that it may be useful to investigate this agent more fully as a potential anticancer agent. Thus, the results presented here suggested that in the future, the copper(II) complexes of Schiff base ligands have good potential as therapeutic agents.

\section{Supplementary material}

CCDC 781723 and 781724 contain the supplementary crystallographic data for 2a and 8a. These data can be obtained free of charge from The Cambridge Crystallographic Data Centre via www.ccdc.cam.ac.uk/data_request/cif.

\section{Acknowledgments}

This research was supported by the Technological Sector Research Programme, Strand 1, under the European Social Fund and the Programme for Research in Third Level Institutes. The research was carried out by the Centre for Pharmaceutical Research and Development (CPRD) and the Centre for Applied Science for Health located at Institutes of Technology, Tallaght, Dublin, and the National University of Ireland, Maynooth, Co., Kildare, Ireland. VRT would also like ITT Dublin for PhD continuation funding.

\section{References}

[1] T.C. White, K.A. Marr, R.A. Bowden, Clin. Microbiol. Rev. 11 (1998) 382.

[2] I.M. Hoepelman, B. Dupont, Int. J. Antimicrob. Ag. 6 (1996) 155

[3] M.V. Kulakarni, G.M. Kulakarni, C.-H. Lin, C.-M. Sun, Curr. Med. Chem. 13 (2006) 2795.

[4] T. Fujioka, S. Teramoto, T. Mori, T. Hosokawa, T. Sumida, M. Tominaga, Y. Yabuuchi, J. Med. Chem. 35 (1992) 3607.

[5] I.V. Ukrainets, S.G. Taran, O.V. Gorokhova, N.A. Marusenko, S.N. Kovalenko, A.V . Turov, N.I. Filimonova, S.M. Ivkov, Chem. Heterocycl. Compd. 31 (1994) 167.

[6] R.G. Kalkhambkar, G.M. Kulkarni, C.M. Kamanavalli, N. Premkumar, S.M.B. Asdaq, C.M. Sun, Eur. J. Med. Chem. 43 (2008) 2178.

[7] I.V. Ukrainets, O.V. Gorokhova, N.A. Jaradat, Chem. Heterocycl. Compd. 42 (2006) 475

[8] (a) P. Angibaud, X. Bourdrez, A. Devine, D.W. End, E. Freyne, Y. Ligny, P. Muller, G. Mannens, I. Pilatte, V. Poncelet, S. Skrzat, G. Smets, J.V. Dun, P.V. Remoortere, M. Veneta, W. Woutersc, Bioorg. Med. Chem. Lett. 13 (2003) 1543;

(b) P. Angibaud, L. Mevellec, C. Meyer, X. Bourdrez, P. Lezouret, I. Pilatte, V. Poncelet, B. Roux, S. Merillon, D.W. End, J.V. Dun, W. Wouters, M. Venet, Eur. J. Med. Chem. 42 (2007) 702;

(c) P. Angibaud, A.K. Saha, X. Bourdrez, D.W. End, E. Freyne, P. Lezouret, G. Mannens, L. Mevellec, C. Meyer, I. Pilatte, V. Poncelet, B. Roux, G. Smets, J.V. Dun, M. Venet, W. Wouters, Bioorg. Med. Chem. Lett. 13 (2003) 4361

[9] C. Xu, L. Yang, A. Bhandari, C.P. Holms, Tetrahedron Lett. 47 (2006) 4885.
[10] D. Ellis, K.L. Kuhen, B. Anaclerio, B. Wu, K. Wolff, H. Yin, B. Bursulaya, J. Caldwell, D. Karanewsky, Y. He, Bioorg. Med. Chem. Lett. 16 (2006) 4246

[11] M.S. Karthikeyan, D.J. Prasad, Bioorg. Med. Chem. 14 (2006) 7482.

[12] B.S. Holla, B. Veerendra, M.K. Shivananda, B. Poojary, Eur. J. Med. Chem. 38 (2003) 759.

[13] K. Singh, M.S. Barwa, P. Tyagi, Eur. J. Med. Chem. 42 (2007) 394.

[14] N. Raman, S. Ravichandran, C. Thangaraja, J. Chem. Sci. 116 (2004) 215.

[15] R. Nair, A. Shah, S. Baluja, S. Chanda, J. Serb. Chem. Soc. 71 (2006) 733.

[16] J. Lv, T. Liu, S. Cai, X. Wang, L. Liu, Y. Wang, J. Inorg. Biochem. 100 (2006) 1888.

[17] T.S. Reddy, N. Rameshwar, B. Bhudevi, A.R. Reddy, Spectrochim. Acta, Part A 73 (2009) 916.

[18] B. Bhudevi, P.V. Ramana, A. Mudiraj, A.R. Reddy, Ind. J. Chem. 48B (2009) 255.

[19] E.A. Mohamed, M.M. Ismail, Y. Gabr, M. Abass, Chem. Papers 48 (1994) 285.

[20] M.A. Phaniband, P.G. Avaji, S.D. Dhumwad, Main Group Chem. 7 (2008) 271

[21] M.A. Phaniband, S.D. Dhumwad, Transition Met. Chem. 32 (2007) 1117.

[22] Z.-C. Liu, B.-D. Wang, Z.-Y. Yang, Y. Li, D.-D. Qin, T.-R. Li, Eur. J. Med. Chem. 44 (2009) 4477.

[23] X. Zhao, P.P.-F. Lee, Y.-K. Yan, C.-K. Chu, J. Inorg. Biochem. 101 (2007) 321.

[24] B.S. Creaven, D.A. Egan, K. Kavanagh, M. McCann, M. Mahon, A. Noble, B. Thati, M. Walsh, Polyhedron 24 (2005) 949.

[25] B.S. Creaven, D.A. Egan, K. Kavanagh, M. McCann, A. Noble, B. Thati, M. Walsh, Inorg. Chim. Acta 359 (2006) 3976.

[26] B.S. Creaven, D.A. Egan, D. Karcz, K. Kavanagh, M. McCann, J. Inorg. Biochem. 101 (2007) 1108.

[27] B. Thati, A. Noble, B.S. Creaven, M. Walsh, K. Kavanagh, D.A. Egan, Eur. J. Pharmacol. 569 (2007) 16

[28] B. Thati, A. Noble, R. Rowan, B.S. Creaven, M. Walsh, M. McCann, D. Egan, K. Kavanagh, Toxicol. in Vitro 21 (2007) 801.

[29] B. Thati, A. Noble, B.S. Creaven, M. Walsh, M. McCann, M. Devereux, K. Kavanagh, D.A. Egan, Eur. J. Pharmacol. 602 (2009) 203.

[30] B.S. Creaven, M. Devereux, D. Karcz, A. Kellett, M. McCann, A. Noble, M. Walsh, J. Inorg. Biochem. 103 (2009) 1196.

[31] B.S. Creaven, M. Devereux, A. Foltyn, S. McClean, G. Rosair, V.R. Thangella, M. Walsh, Polyhedron 29 (2010) 813.

[32] Q. Zhang, Y. Chen, Y.Q. Zheng, P. Xia, Y. Xia, Z.Y. Yang, K.F. Bastow, S.L. MorrisNatschke, K.-H. Lee, Bioorg. Med. Chem. 11 (2003) 1031.

[33] APEX2 Version 2.1 Bruker AXS Inc., Madison, Wisconsin, USA, 2004-2006.

[34] G.M. Sheldrick, Acta Cryst., Sect. A 64 (2008) 112.

[35] National Committee for Clinical Laboratory Publication, Villanova, PA, M27-P, 1979.

[36] T. Mosmann, J. Immunol. 65 (1983) 55.

[37] A. Golcu, M. Tumer, H. Demirelli, R.A. Wheatley, Inorg. Chim. Acta. 358 (2005) 1785.

[38] S.M. Ben-saber, A.A. Maihub, S.S. Hudere, M.M. El-ajaily, Microchem. J. 81 (2005) 191.

[39] 40.M.S. Refat, I.M. El-Deen, H.K. Ibrahim, S. El-Ghool, Spectrochim. Acta, Part A 65 (2006) 1208.

[40] K.K. Upadhyay, A. Kumar, S. Upadhyay, P.C. Mishra, J. Mol. Struct. 873 (2008) 5.

[41] M.L. Creber, K.G. Orrell, A.G. Osborne, V. Sik, S.J. Coles, D.E. Hibbs, M.B. Hursthouse, Inorg. Chim. Acta 299 (2000) 209.

[42] N. Raman, S. Ravichandran, C. Thangaraja, J. Chem. Sci. 116 (4) (2004) 215.

[43] K. Serbest, A. Ozen, Y. Unver, M. Er, I. Degirmencioglu, K. Sancak, J. Mol. Struct. 922 (2009) 1.

[44] Kiran Singh, Manjeet Singh Barwa, Parikshit Tyagi, Eur. J. Med. Chem., 2006, 1.

[45] P. Kopel, J. Kamenıcek, V. Petrıcek, A. Kurecka, B. Kalinska, J. Mrozinski, Polyhedron 26 (2007) 535

[46] N.C. Pramanik, S. Bhattacharya, Transition Met. Chem. 23 (1998) 429.

[47] A. Majumder, G.M. Rosair, A. Mallick, N. Chattopadhyay, S. Mitra, Polyhedron 25 (2006) 1753

[48] M. Shebl, Spectrochim. Acta, Part A 70 (2008) 850.

[49] S. Chnadra, L.K. Gupta, Spectrochim. Acta, Part A 62 (2005) 307

[50] P. Biswas, S. Dutta, M. Ghosh, Polyhedron 27 (2008) 2105.

[51] R.M. Issa, A.M. Khedr, H.F. Rizk, Spectrochim. Acta, Part A 62 (2005) 621.

[52] D. Maiti, H. Paul, N. Chanda, S. Chakrabort, B. Mondal, V.G. Puranik, G.K. Lahiri, Polyhedron 23 (2004) 831.

[53] R. Gup, B. Kırkan, Spectrochim. Acta, Part A 62 (2005) 1188.

[54] R. Gup, B. Kirkan, Spectrochim. Acta, Part A 64 (2006) 809.

[55] A. Sousa, M.R. Bermejo, M. Fondo, A. Garcia-Deibe, A. Sousa-Pedrares, O. Piro, New J. Chem. 25 (2001) 647.

[56] E. Prenesti, S. Berto, P.G. Daniele, Spectrochim. Acta A 59 (2003) 201.

[57] S. Pang, Y. Liang, Spectrochim. Acta A 57 (2001) 435.

[58] P. Mayer, T.I.A. Gerber, B. Buyambo, A. Abrahams, Polyhedron 28 (2009) 1174.

[59] A. Valent, M. Melnik, D. Hudecova, B. Dudova, R. Kivekas, M.R. Sundberg, Inorg. Chim. Acta 340 (2002) 15

[60] A.L. Iglesias, G. Aguirre, R. Somanathan, M. Parra-Hake, Polyhedron 23 (2004) 3051.

[61] W.N. Wallis, S.C. Cumming, Inorg. Chem. 13 (1974) 991.

[62] S. Higashiya, K.K. Banger, S.C. Ngo, P.N. Lim, P.J. Toscano, J.T. Welch, Inorg. Chim. Acta 351 (2003) 291.

[63] Z.-X. Li, X.-L. Zhang, X.-H. Pu, Acta Cryst., Sect. E 64 (2008) 202.

[64] A.K. Sharma, R. Mukherjee, Inorg. Chim. Acta 361 (2008) 2768.

[65] M. Geraghty, V. Sheridan, M. McCann, M. Devereux, V. McKee, Polyhedron 18 (1999) 2931.

[66] S. Abuskhuna, M. McCann, J. Briody, M. Devereux, V. McKee, Polyhedron 23 (2004) 1731.

[67] B. Sarkar, G. Bocelli, A. Cantoni, A. Ghosh, Polyhedron 27 (2008) 693. 
[68] M. Espinal, J. Pons, J. Garcia-Anton, X. Solans, M. Font-Bardia, J. Ros, Inorg. Chim. Acta 361 (2008) 2648.

[69] K. Sundaravel, E. Suresh, M. Palaniandavar, Inorg. Chim. Acta 362 (2009) 199

[70] A.L. Spek, J. Appl. Cryst. 36 (2003) 7.

[71] M.C. Etter, J.C. MacDonald, J. Bernstein, Acta Cryst., Sect. B 46 (1990) 256
[72] B. Zurowska, A. Brzuszkiewicz, Polyhedron 27 (2008) 1623.

73] A. Kochel, Inorg. Chim. Acta 362 (2009) 1379.

[74] B.K. Maiti, K. Pal, S. Sarkar, Dalton Trans., 20081003.

[75] A. Noble, R. Rowan, B.S. Creaven, M. Walsh, M. McCann, D. Egan, K. Kavanagh, Toxicol. in Vitro 21 (2007) 801. 